\title{
Stephanoscyphus eumedusoides n. spec. (Scyphozoa, Coronatae), ein Höhlenpolyp mit einem neuen Entwicklungsmodus
}

\author{
B. WERNER \\ Biologische Anstalt Helgoland (Zentrale); \\ Hamburg 50, Bundesrepublik Deutschland
}

\begin{abstract}
Stephanoscyphus eumedusoides n. spec. (Scyphozoa, Coronatae), a cave-living polyp with a new mode of development. The new solitary scyphopolyp Stephanoscyphus eumedusoides was detected in submarine caves of the rocky shore near Marseille (Mediterranean Sea), where it lives attached to colones of corals at a depth of 2-3 m to $40-50 \mathrm{~m}$. The new mode of development has been described briefly in an earlier paper in which the polyp was given the preliminary name Tesseroscyphus eumedusoides (WERNER 1971a) but by a more detailed investigation it proved to belong to the genus Stephanoscypbus. The essential characteristics of the peridermal tube and the soft body are outlined. Most of them are not sufficient to identify significantly the new species. Identification was possible by the observations on the new mode of development by sessile medusoids which is unique in the order Coronatae and the class Scyphozoa. The medusoids originate in the normal way by the process of strobilation. But other than in the known scyphozoan development by which freeswimming young medusae are produced, the medusoids remain connected with each other and the basal residue of the polyp's body within the peridermal tube. Development, morphology and anatomy of the medusoids are described. As they exhibit essential medusan characteristics they belong to the type of eumedusoid. On the other hand, there are remarkable signs of reduction due to progressive steps of evolution. The hermaphroditic medusoids become mature and reproduce within the polyp's tube. The fertilized eggs develop within the gastric cavity of the medusoids into free-swimming planulae which are released by the chain of degenerating and dying medusoids being pushed out of the tube by the regenerating polyp. After a planktonic period of several weeks, the planula attaches to a substratum in the normal way and undergoes development into the young polyp. This is described briefly. The cnidom of the polyp, the medusoid, and the planula consists of holotrichous haplonemes and heterotrichous microbasic euryteles. The vertical and horizontal distribution, and some details of the ecology are outlined according to the present state of knowledge. Because the new species has been collected and found only in submarine caves it is considered to be a true cave-living animal. Its diagnosis is given.
\end{abstract}

\section{EINLEITUNG}

In einer kurzen Mitteilung wurde ein neuer solitärer Scyphopolyp beschrieben (Werner 1971a, vgl. Werner 1971b), der wie Stephanoscyphus eine vollständige Peridermröhre besitzt, sich aber von den anderen Formen dieses Genus und von allen an- 
deren metagenetischen Scyphozoen durch seine Entwicklung unterscheidet. Er stammt aus dem Felslitoral der Küste von Marseille, wo er in submarinen Höhlen stellenweise häufig gefunden wird. Bei dem neuen Polypen entstehen die Medusenanlagen in der für Scyphozoen typischen Weise durch Strobilation. Die in der Röhre durch Querteilung des Polypenkörpers erzeugte Strobilationskette löst sich jedoch nicht in freischwimmende Ephyren auf, wie das in der Normalentwicklung der metagenetischen Scyphozoen der Fall ist, deren Medusen bekanntlich erst während einer längeren pelagischen Phase vom Larvalstadium zur vollen Größe und Geschlechtsreife heranwachsen. Vielmehr bleibt bei dem Marseiller Polypen die ganze Strobilationskette mit dem basalen Restkörper und bleiben alle Medusenanlagen miteinander in fester Verbindung; sie werden in diesem Zustand ohne Nahrungszufuhr von außen geschlechtsreif und pflanzen sich fort. Durch die Strobilation entsteht also in der Röhre des Polypen eine zusammenhängende Kette von $\mathrm{Medu}$ s oiden. Die Keimzellen werden in den Gastralraum der Medusoide abgegeben, wo ihre Entwicklung zur begeißelten Planula in der normalen Weise abläuft. Sie werden am Ende der Fortpflanzungsphase frei, wenn der regenerierende Polyp die Strobilationskette aus der Röhre herausschiebt, die sich in die Einzelmedusoide auflöst. Diese sterben schließlich ab und zerfallen.

Damit war bei metagenetischen Scyphozoen zum ersten Male ein eindeutiger Fall der Reduktion der Medusengeneration bekannt geworden: die Medusen werden angelegt und fortpflanzungsfähig, werden aber nicht mehr frei, sondern bleiben mit dem Polypen innerhalb der Röhre in fester Verbindung, bis sie ihre Funktion erfüllt haben und absterben. Vorher lagen mit den Untersuchungen von KomaI (1935) und KomaI \& Tokuoka (1939) zwar Hinweise auf das Vorkommen von Medusoiden bei der in Japan endemischen Art Stephanoscyphus racemosus vor, doch konnte damals die Entwicklungsgeschichte dieser Art nicht vollständig geklärt werden. Das ist erst neuerdings gelungen, und es konnte gezeigt werden, daß die japanische Art tatsächlich ebenfalls Medusoide erzeugt (WERNER 1973).

Die Reduktion der freischwimmenden Medusengeneration zu sessilen Gonophoren ist bekanntlich bei den Hydrozoen nicht selten. KüHN (1913) hat die verschiedenartigen Reduktionserscheinungen der Gonophoren bei den Hydroidpolypen eingehend untersucht und eine evolutionistische Entwicklungsreihe der progressiven Reduktion aufstellen können, deren Stadien als Eumedusoide, Heteromedusoide, Kryptomedusoide und Sporosacs in die Literatur eingegangen sind. Wie sich unmittelbar ergab, steht einer partiellen Übernahme der Terminologie für den vorliegenden Fall der Medusenreduktion bei einem Scyphopolypen nichts im Wege. Da es sich bei seinen Strobilationsprodukten um Gebilde mit deutlich erhaltenem Medusencharakter handelt, können sie in Analogie zur ersten Stufe der Reduktionsreihe der Hydroiden als Eumedusoide bezeichnet werden.

Im Vergleich zu den scheibenförmigen Ephyren und ebenso zu den erwachsenen Medusen der meisten anderen Coronaten haben die Eumedusoide des neuen Polypen eine ungewöhnliche Morphologie, da sie ausgesprochen glodkenförmig gebaut sind. Außerdem fehlt ihnen die typische Ringfurche zwischen Zentralteil des Schirms und dessen Randbezirken, die hinsichtlich der Organe des Schirmrandes, vor allem mit dem Fehlen von Statocysten weitere Reduktionserscheinungen aufweisen. Wesentlich ist auch, daß die Zahl der Gonaden auf 4 reduziert ist. Bei der Erstbeschreibung des für 
Scyphozoen neuen Entwicklungsganges erschienen mir diese Unterschiede so erheblich, daß ich die Errichtung des neuen Genus Tesseroscyphus für notwendig hielt. In dieser Auffassung wurde ich auch dadurch bestärkt, daß ich bei der Suche nach einer Art mit freischwimmenden Medusen, die als Vorfahr des neuen Polypen hätte gelten können, in Tessera princeps HaEckel, 1880, auf eine Scyphomeduse gestoßen war, deren wesentliche Merkmale, nämlich Glockenform des Schirms, Fehlen der Ringfurche, Oktomerie des Schirmrandes, Fehlen von Statocysten, Vierzahl der Gonaden, die Annahme verwandtschaftlicher Beziehungen nahelegten.

Die weiteren Beobachtungen über die Entwicklungsgeschichte des Marseiller Polypen, der sich nunmehr über fünf Jahre in Laboratoriumskultur befindet, ferner vergleichende Untersuchungen über die Entwicklung der erwähnten japanischen Form Stephanoscyphus racemosus haben mich jedoch zu einer Uberprüfung der Erstbefunde und zu einer anderen Beurteilung der Reduktionserscheinungen veranlaßt, die eine Revision der bisherigen systematischen Bewertung zur Folge haben. Die wichtigste Beobachtung, die die Errichtung eines neuen Genus überflüssig macht, betrifft die Entwicklung und Struktur des Schirmrandes der Medusoide. Es hat sich gezeigt, daß die Organe des Schirmrandes bei den jungen, in der Entwicklung begriffenen und in der Polypenröhre befindlichen Medusoiden in der gleichen Weise angelegt werden können wie bei den Ephyren von Arten mit freien Medusen. Sie lassen dann die gleiche Aufteilung des Schirmrandes in 8 Randlappenpaare erkennen, wie sie für die Ephyren des Genus Nausitbo $\ddot{e}$ typisch sind. Andererseits wurde beobachtet, daß die Bildung der Randlappen nicht bei allen Medusoiden in einheitlicher Weise erfolgt, daß sie vielmehr variieren kann; sie weisen dann einen unterschiedlichen Grad der Rückbildung auf. Hinzu kommt, daß der Entwicklungszustand des Schirmrandes bei den älteren Medusoiden, die vom Polypen aus der Röhre herausgeschoben werden, dann vor dem Zerfall stehen und oft schon geschrumpt sind, vielfach nicht mehr eindeutig erkennbar ist.

In jedem Fall treten bei den Medusoiden Reduktionserscheinungen des Schirmrandes mit Regelmäßigkeit auf. Sie sind jedoch nach den neuen Beobachtungen nicht als primär einfach, das heißt als primitiv, sondern müssen als sekundär vereinfacht und wie die übrigen Reduktionserscheinungen als Ergebnis einer progressiven Evolution gedeutet werden. Daher ist anzunehmen, daß der neue Polyp von Vorfahren abstammt, die normale, scheibenförmige Ephyren und freilebende Medusen des Nausithoë-Typs gehabt und wahrscheinlich diesem Genus angehört haben. Wegen ihrer unterschiedlichen Entwicklung und wegen des Fehlens freier Medusen kann die neue Art jedoch nicht in das Genus Nausithoë selbst eingeordnet werden; vielmehr gehört sie in das Genus Stephanoscypbus und erhält deswegen den Namen S.eumedusoides, wie schon früher angekündigt worden ist (WERNER 1973).

Der genaueren Beschreibung der Morphologie und Entwicklung der neuen Art dient der folgende Beitrag; er ergänzt damit die kurze Erstbeschreibung, die zunächst nur mit dem ungewöhnlichen Entwicklungsgang bekannt machen sollte. So ist es jetzt auch möglich, die noch fehlende Artdiagnose einzuschließen. 


\section{MATERIAL UND METHODE}

Die Entdeckung der neuen Art Stephanoscyphus eumedusoides ist Herrn Dr. Zibrowius, Marseille, zu verdanken, der bei Tauchuntersuchungen über die sessile Fauna untermeerischer Höhlen der Felsküste von Marseille das vereinzelte, stellenweise auch häufige Vorkommen von solitären Polypen festgestellt hatte, die nach Form und Struktur der Peridermröhren dem Genus Stephanoscyphus angehören mußten und der Art S. simplex zugeordnet wurden (Zrbrowrus 1968). Die Genus-Diagnose war bei der Untersuchung des konservierten Materials leicht zu bestätigen. Es handelte sich um eine Form mit kräftiger, braungelb gefärbter Röhre, die meist an Korallenstöckchen der Arten Leptopsammia pruvoti, Holoplangia durotrix und Coenocyathus dobrni angeheftet waren. Die Größe der Polypen war gering und betrug meist nur 5 bis $10 \mathrm{~mm}$; immerhin war die Mehrzahl groß genug, daß sie mit bloßem Auge erkennbar war.

Die weitere Untersuchung des konservierten Materials bestätigte die für viele Stephanoscyphus-Arten zutreffende Erfahrung, daß eine einwandfreie Identifizierung nach Strukturmerkmalen der Röhre und des stark kontrahierten Weichkörpers nicht möglich ist. Ferner hatte sich schon vorher gezeigt, daß sich unter den beiden in der Literatur beschriebenen solitären Arten S. simplex KIRKPATRICK, 1890, und S. corniformis KomaI, 1936, eine ganze Anzahl verschiedener Arten verbirgt, und daß nur die genaue Kenntnis des ganzen Lebenszyklus die sichere systematische Einordnung zuläßt. So erwies es sich als notwendig, für die nähere Untersuchung des Marseiller Polypen lebendes Material zu erhalten. Das besondere Interesse an dieser Form lag darin begründet, daß aus dem Mittelmeer bislang eine solitäre Form nur durch Funde aus gröBeren Wassertiefen bekannt war (Lo Bianco 1903).

Durch den persönlichen Taucheinsatz von Herrn Dr. Zibrowius gelang es, im Oktober 1969 ausreichende Mengen von lebenden Polypen zu erhalten, die den Transport in Thermogefäßen gut überstanden und in Laboratoriumsdauerkultur genommen werden konnten. Die Daten der Fundchronik sind in der Tabelle 1 zusammengestellt. Während der Zuchtperiode des Winters und Frühjahrs 1969-70 begannen die Polypen zu strobilieren und ließen dann den neuen Entwicklungsmodus erkennen. Dabei stellte sich bald heraus, daß die Strobilation ein temperaturabhängiger Prozeß ist, der im Laboratorium bei Temperaturen von 15 bis $17^{\circ} \mathrm{C}$ im langfristigen Wechsel mit einer Erholungsphase regelmäßig erfolgt. Die obere Grenztemperatur lag bei etwa $22^{\circ} \mathrm{C}$. Diese Erfahrung legte den Versuch nahe, die Strobilationsperiode bei Polypen am Fundort zu erfassen. Bei dem reichhaltigen Material vom Oktober 1969 war keine einzige Strobila beobachtet worden. $\mathrm{Da}$ an der Nordküste des Mittelmeeres die Wassertemperaturen in den Tiefenstufen von $O$ bis $50 \mathrm{~m}$ während des Winters auf 12 bis $10^{\circ} \mathrm{C}$ absinken, da ferner in einer Wassertiefe von $20 \mathrm{~m}$ im Frühjahr bei steigenden Temperaturen die kritische Grenze von etwa $15^{\circ} \mathrm{C}$ im Mai bis Juni erreicht wird (BHAud et al. 1967, vgl. MrNas 1971), konnte für den Anfang Juni das Vorkommen von Strobilae an den Fundorten vorausgesagt werden, falls der Eintritt der Strobilation wirklich von der Temperatur abhängig ist.

Der Erfolg hat diese Erwartung bestätigt; bei der gezielten Probenentnahme am 10.6. 1970 zeigte sich, daß tatsächlich ein nicht unerheblicher Teil der gesammelten Polypen (ca. 11\%) in Strobilation begriffen war und daß weitere Strobilationen kurz 
Tabelle 1

Stephanoscyphus eumedusoides n. spec. Fundliste für das westliche Mittelmeer und die Atlantikküste (Portugal)

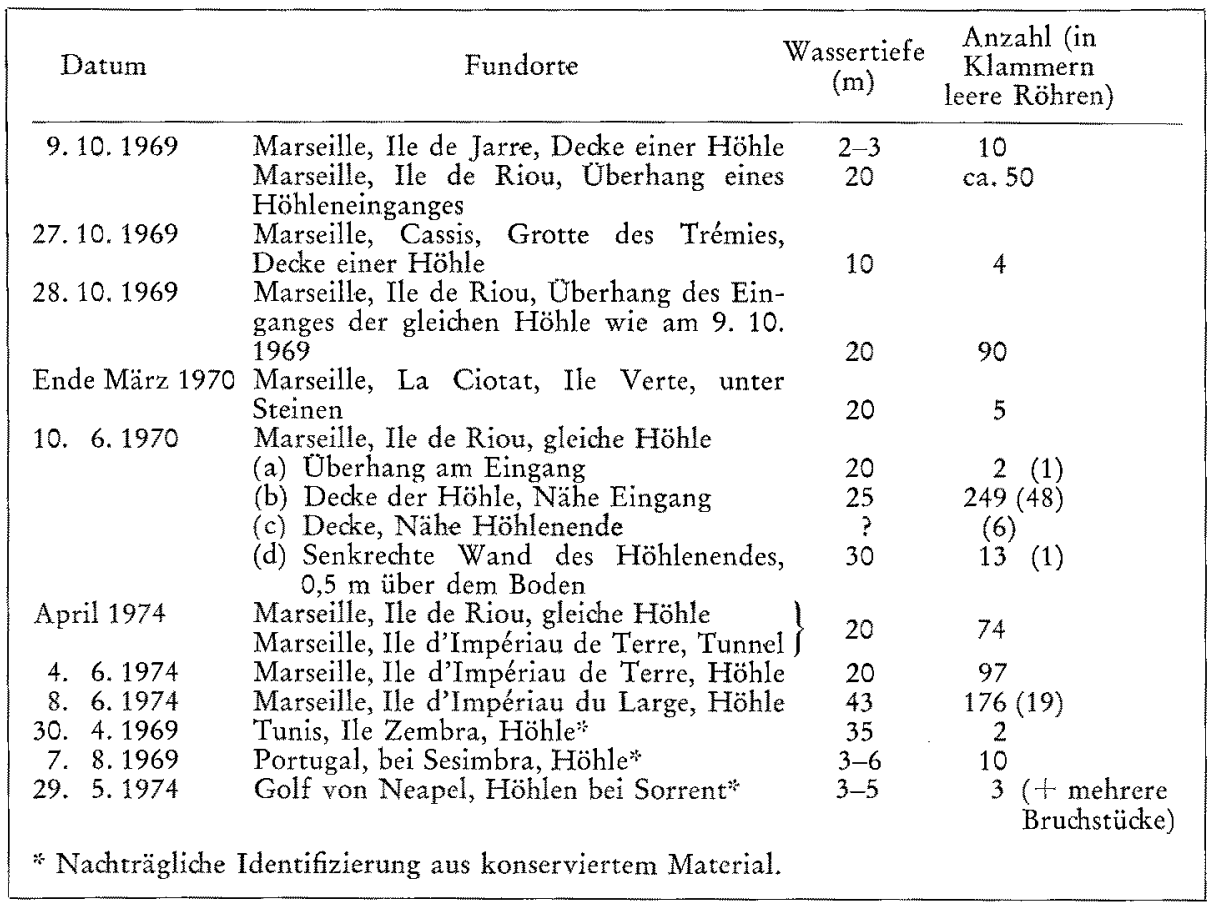

darauf im Laboratorium auftraten. Übereinstimmende Ergebnisse wurden im Juni 1974 erzielt, als weiteres Lebendmaterial in anderen submarinen Höhlen bei Marseille gesammelt und anschließend untersucht wurde.

Beim Sammeln wurden vom Taucher die Korallenstöckchen von der Decke und den Wänden der submarinen Höhlen mit Hammer und Meißel abgelöst und sofort an Bord des Schiffes auf das Vorkommen von Polypen untersucht und sortiert. Diese wurden dann anschließend in Laboratorium mit Hilfe des Stereomikroskopes vom gröberen Ansatzmaterial befreit. Die Polypen wurden dabei nach Möglichkeit mit einem kleinen Stück des natürlichen Ansatzmaterials belassen, um sie nicht zu beschädigen und um die basale Haftscheibe unverletzt zu lassen. Es zeigte sich, daß zahlreiche Polypen tief in den. Höhlungen und Spalten der Korallenstöckchen angeheftet waren, so daß es nicht immer gelang, die Röhren völlig unbeschädigt mitsamt den Haftscheiben abzulösen. Doch bestätigte sich die an anderen Arten gemachte Erfahrung, daß der Verlust der Haftscheibe oder auch eines kleineren Teiles der ganzen Basis keine nennenswerte Schädigung für den Polypen darstellt. Er verschließt die akzidentelle untere Offnung mit einer Peridermquerwand und lebt auf dem Boden der Kulturschale in normaler Weise weiter.

Die bei den langfristigen Kulturversuchen angewandten Methoden wurden bereits früher beschrieben (WeRner 1968, vgl. WernER 1971b). Hinsichtlich der Ernährung 
ist zu ergänzen, daß die individuelle Fütterung der Polypen mit Stückchen frischer Mitteldarmdrüse von Mytilus edulis und die zweigliederige Kultur mit dem Harpacticiden Tisbe holothuriae die besten Kulturerfolge brachten. Das verwendete Seewasser stammte aus der Umgebung von Helgoland und wurde stets in Glasballons transportiert und aufbewahrt. Durch Zufügen entsprechender Mengen von $\mathrm{NaCl}, \mathrm{MgSO}_{4}$, $\mathrm{CaCl}_{2}, \mathrm{KCl}, \mathrm{Na}_{2} \mathrm{CO}_{3}$ wurde es auf einen annähernd konstanten Wert von $34,50 \%$ eingestellt. Für die vorläufige histologische Untersuchung wurden die Polypen und Strobilae mit 7,5\%-Lösung von $\mathrm{MgCl}_{2}$ in Aqua dest. betäubt; anschließend Fixierung mit Bouin und Einbettung in Paraffin. Kernfärbung mit Delafield's Hämatoxylin, Nachfärbung mit Eosin.

\section{BEFUNDE}

\section{Die Morphologie des Polypen}

Peridermröhre und Weichkörper von Stephanoscyphus eumedusoides weisen einige Unterschiede gegenüber anderen Arten des Genus auf, wie im einzelnen zu zeigen ist. Doch reichen die unterschiedlichen Strukturmerkmale des Polypen allein nicht aus, ihn als neue Art zweifelsfrei abzugrenzen. Seine Sonderstellung ist vielmehr durch die vollständige Kenntnis seines Entwicklungsganges zu begründen, der daher auch in der Artdiagnose berücksichtigt werden muß.

\section{Die Peridermröbre}

Ein charakteristisches Merkmal ist der solitäre Habitus, da niemals ein Polyp angetroffen wurde, der auch nur Spuren einer Verzweigung hätte erkennen lassen. Der Weichkörper wird von der wohlentwickelten Peridermröhre vollständig eingeschlossen, so daß aus deren oberer Mündung nur der Kopfteil mit der Tentakelkrone herausschaut (Abb. 1-3,6). Der sich verjüngende Basalteil ist am Substrat mit einer kleinen Haftscheibe befestigt, welche die für Stephanoscypbus übliche Form hat. Ihr Umriß ist allerdings oft unregelmäßig, da er von der Form und Beschaffenheit der Ansatzstelle abhängt. Im Normalfall hat die Haftscheibe einen rundlichen oder ovalen Umriß und ähnelt im Jugendzustand einer einfachen glattwandigen niedrigen Kuppel, von der sich die Röhre schornsteinförmig erhebt. Durch sekundäre Wachstumserscheinungen wird der Rand später unregelmäßig gelappt, und der ursprünglich einheitliche Innenraum wird durch radiale Vertikalwände so aufgegliedert, daß unregelmäßig geformte niedrige Seitenkammern entstehen, die zur Mitte und zum Röhrenlumen geöffnet sind. Der Durchmesser des basalen Röhrenteils dicht über der Haftscheibe ist annähernd konstant und beträgt $0,2 \mathrm{~mm}$. Der Durchmesser der Haftscheibe ist wegen der oft unregelmäßigen Beschaffenheit schwer zu ermitteln. Er beträgt bei jungen, aus der Planula gezüchteten Polypen, die sich auf ebener Unterlage (Boden der Kulturschale) angeheftet hatten, im Durchschnitt $0,6 \mathrm{~mm}$.

Im Vergleich zu anderen Arten muß für S. eumedusoides die besonders kräftige Ausbildung der Röhre hervorgehoben werden, was vor allem für die untere Röhren- 
hälfte zutriff. Hier hat die Röhre entsprechend eine relativ dicke Wandung und eine kräftig dunkel- bis mittelbraune Färbung, während der obere Röhrenteil eine hellbraune bis gelbe Färbung aufweist. Bei einer Röhre von $22,2 \mathrm{~mm}$ Länge hatte die Wandung des Basalteils eine Dicke von $0,021 \mathrm{~mm}$ und nahm im unteren Drittel noch auf $0,026 \mathrm{~mm}$ zu. Die Wanddicke betrug dicht unterhalb der sehr zarten Röhrenmündung
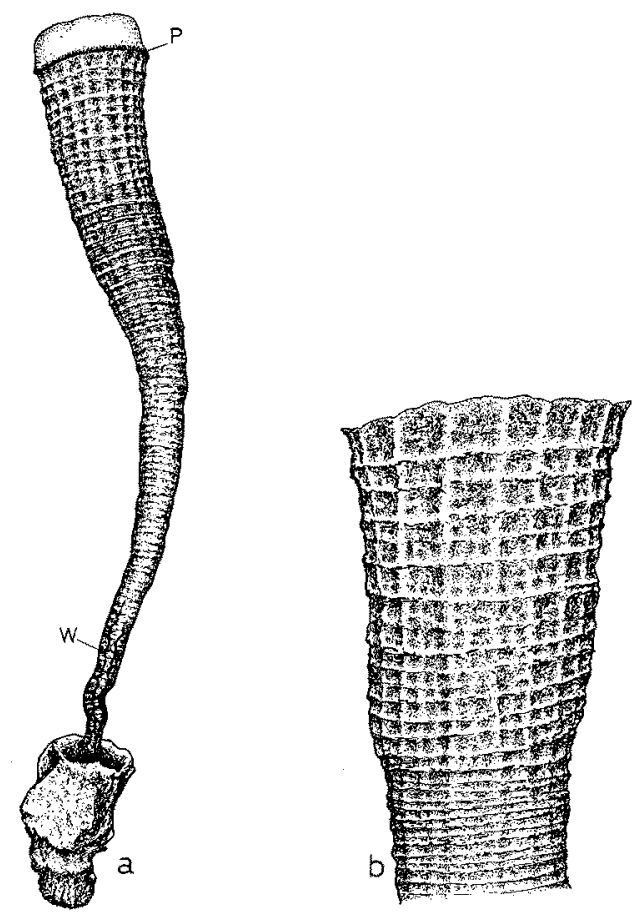

Abb. 1 a, b: Stephanoscyphus eumedusoides n. spec., lebender Polyp, dessen Weichkörper bis auf den Kragen kontrahiert ist. Zu beachten ist in $b$ die etwas größere Breite und veränderte Oberflächenstruktur des oberen Röhrenteils, die auf bessere Wachstumsbedingungen in der Kultur zurückzuführen sind, ferner in a die lamellenartigen Wandverstärkungen im Basalteil. P Pigmentring, W Wandverstärkungen. Länge der Röhre $8,6 \mathrm{~mm}$, oberer Durchmesser 1,45 mm.

(Gesammelt am 28.10. 1969; Zeidinung: F. HecKmANn, 12. 5. 1970)

$0,002 \mathrm{~mm}$. Die ausgesprochen kräftige Färbung der Röhre ist wahrscheinlich durch die stärkere Einlagerung von Pigment bedingt, worauf das Vorhandensein eines Pigmentringes am unteren Rand des Kragens, das heißt im Bereich der Bildung der Röhrensubstanz, hinweist (vgl. p. 445).

Die Länge der Röhre ist vom Alter und von den Wachstumsbedingungen abhängig und starken individuellen Schwankungen unterworfen. Das am 28.10.1969 gesammelte und im März/April 1970 gemessene Material hatte eine durchschnittliche Größe von 5 bis $10 \mathrm{~mm}$; das Maximum betrug $16 \mathrm{~mm}$. Bei dem gleichen Material betrug die durchschnittliche Länge Ende August 1972, also nach fast dreijähriger Kulturzeit, 7 bis $15 \mathrm{~mm}$, bei einem Maximum von 26,5 mm. Der Durchmesser der Röhrenmündung be- 
trug 0,96 bzw. $1,63 \mathrm{~mm}$. Nach allen Beobachtungen verlangsamt sich das Wachstum bei alten Polypen und dürfte eine maximale Länge von 25 bis $30 \mathrm{~mm}$ nicht überschreiten.

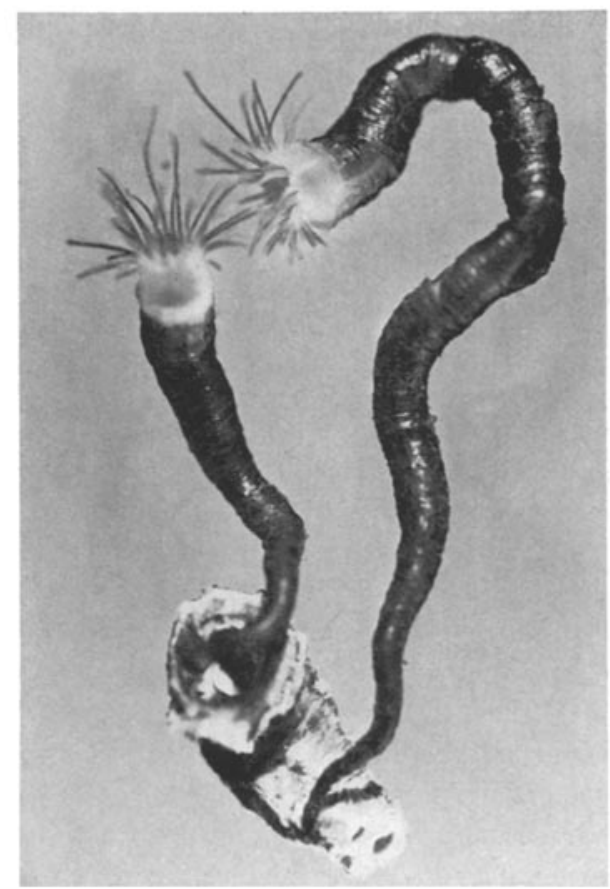

Abb. 2

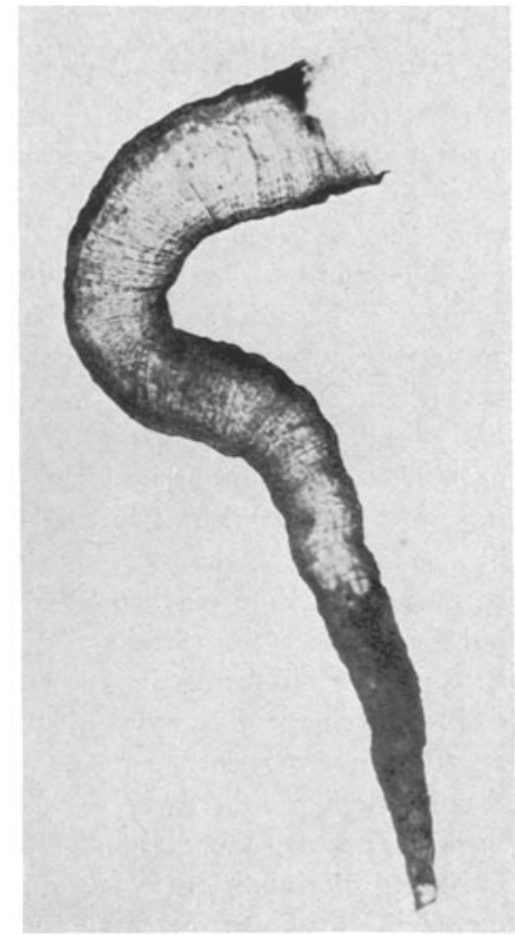

Abb. 3

Abb. 2: Stephanoscyphus eumedusoides n. spec., Auflichtphoto lebender Polypen. Die unregelmäßig gekrümmten Röhren sind häufig bei Polypen anzutreffen, die an Korallenstöckchen angeheftet sind. $(1: 6)$

Abb. 3: Stephanoscyphus eumedusoides n. spec., Photo einer beim Fang leeren Röhre. Die Oberflächenstruktur mit schmalen, dicht aufeinanderfolgenden Querringen und schwach ausgeprägter oder fehlender Längsstreifung ist charakteristisch für fangfrisches Material. $(1: 14)$

Die Form der Röhre ist bei frischgesammelten Polypen relativ schlank, und es ist eine kontinuierliche langsame Erweiterung von der Basis bis zur oberen Mündung zu verzeichnen. Um so mehr fiel auf, daß sich der Durchmesser des oberen Röhrenteils bei gut ernährten Kulturexemplaren relativ schneller vergrößerte, so daß sich an dieser Formänderung der Einfluß der unterschiedlichen Lebensbedingungen direkt ablesen ließ (Abb. 1b). Diese Beobachtung spricht dafür, daß S. eumedusoides nach der Form der Röhre eher zur S. simplex-Gruppe gehört als zur S. corniformis-Gruppe, da die letztere Art nach KRAMp (1959) eine etwas schlankere Röhre besitzt. Auf die unterschiedlichen Formverhältnisse der Röhren bei solitären Arten von Stephanoscyphus, für die nach Kramp als quantitativ erfaßbare Größe der Quotient (Durchmesser der 
Röhrenmündung : Gesamtlänge der Röhre) angegeben werden kann, wird in einer späteren Arbeit näher einzugehen sein. Bei dem erwähnten Lebendmaterial von $S$, entmedusoides, das Ende August 1972 gemessen wurde und eine fast dreijährige Kulturzeit hinter sich hatte, betrug dieser Quotient $\frac{D}{L}=0,11$.

Weiterhin ist bemerkenswert, daß die Polypen oft unregelmäßig gekrümmte Röhren hatten, wodurch sie sich gleichfalls von anderen Arten unterscheiden lassen, die meist gerade bis schwach hornförmig gekrümmt sind. Die unregelmäßige Röhrenkrümmung bei S. eumedusoides (Abb. 2) ist jedoch zweifellos sekundärer, zufälliger Natur und hängt mit den Wachstumsbedingungen am natürlichen Standort zusammen. Wie erwähnt, wurden die meisten Polypen an Korallenstöckchen gefunden. Beim Ablösen zeigte sich, daß sich die Planula-Larven mit Vorliebe in den feinen Ritzen und Spalten an der Skelettbasis der Stöckchen angeheftet haben mußten, so daß die sich aus ihnen entwickelnden Polypen ihre Wachstumsrichtung den örtlichen Gegebenheiten anpassen und sie oft ändern mußten. Hinzu kommt, daß die Polypen, die an der Höhlendecke angeheftet sind, nicht frei nach oben wachsen können, wie das ihrer normalen Wachstumsrichtung entspricht. Die langjährigen Kulturversuche mit zahireichen Polypen haben bestätigt, daß das erwähnte unregelmäßige Formwachstum nicht als normal zu betrachten ist. Zum gleichen Ergebnis führte die Aufzucht von Jungpolypen aus Planulae, die die typische, schwach hornförmige Krümmung aufwiesen. Die praktische Erschwerung einwandfreier Längenmessungen bei dem Höhlenmaterial läßt sich nur dadurch einigermaßen ausgleichen, daß die Krümmungen ausgemessen und die Teilwerte addiert werden.

Die äußere Oberfläche der Röhre weist zwar die für Stephanoscyphus typische Struktur mit deutlichen Querringen und feinen Längsstreifen auf; doch bestehen auch in diesem Merkmal Unterschiede zwischen den frisch gesammelten und den über längere Zeit kultivierten Exemplaren. Bei den ersteren sind nämlich die Querringe of sehr schmal, was offenbar auf langsames Wachstum zurïdzzuführen ist (Abb. 3). Dadurch tritt die feine Längsstreifung weniger deutlich in Erscheinung oder ist überhaupt nicht zu erkennen. Im Gegensatz dazu führt das schnellere Wachstum bei den Kulturtieren zur Ausbildung breiterer Querringe, bei denen dann auch die Längsstreifung deutlich ausgeprägt ist. In Abbildung $1 \mathrm{~b}$ ist die Anderung der Oberflächenstruktur bei einem Kulturexemplar deutlich. Daher gehört auch bei S. eumedusoides die Quer- und Längsstreifung zum Grundmuster der Oberflächenstruktur der Peridermröhre.

Die Innenseite des größten Teiles der Röhrenwandung ist glatt. Nur im Basalteil treten Zahnbildungen und unregelmäßige, lamellenähnliche Wandverstärkungen auf. Wie früher beschrieben wurde (Kramp 1959, Werner 1967, Chapman \& Werner 1972) muß das Vorhandensein von mehreren Zahnkränzen auf der Innenseite der Röhrenwandung als für die meisten Arten von Stephanoscypbus typisch gelten. Bei ihnen besteht ein solcher Zahnkranz meist aus 4 größeren perradialen und 4 kleineren interradialen Zähnen, die über Kreuz angeordnet sind und im gleichen Niveau liegen. Bei der Röhre von $S$. eumedusoides findet sich jedoch meist nur ein einziger Zahn im Basisteil, der eine perradiale Lage hat (Abb. 4, 5a, b). Bei der vergleichenden Prüfung eines größeren Materials zeigte sich, daß nur in wenigen Fällen 2 solcher Einzelzähne vorhanden und daß sie dann senkrecht übereinander angeordnet waren. In anderen Fällen 
fehlten Zahnbildungen vollständig. Das Fehlen von Zahnkränzen ist als sekundäres Merkmal zu betrachten, und man muß annehmen, daß S. eumedusoides von Vorfahren abstammt, bei denen sie vollständig ausgebildet waren.

Ferner lassen sich im Basisteil der Röhre häufig Peridermstrukturen beobachten, die bei anderen Arten bisher nicht angetroffen wurden. Sie bestehen aus lamellenartigen Wandverstärkungen, die schon äußerlich bei der Betrachtung im Auflicht gut er-

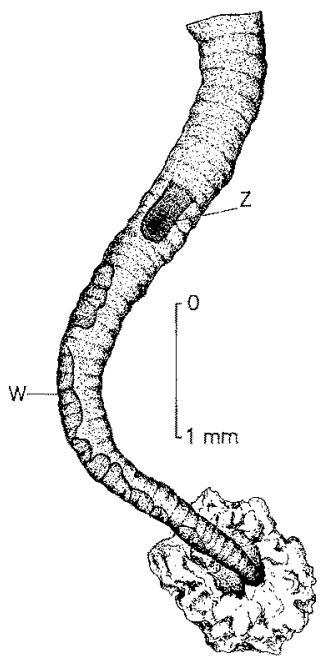

$\mathrm{Abb}, 4$
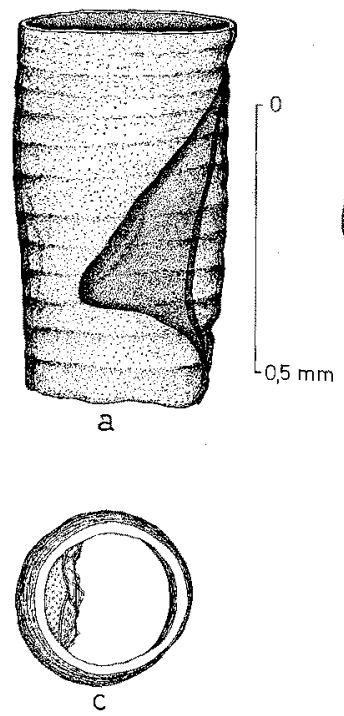

Abb. 5
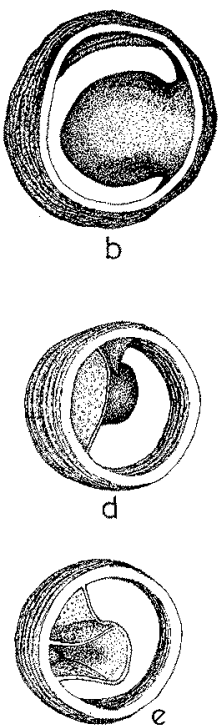

Abb. 4: Stephanoscyphus eumedusoides n. spec., Basalteil einer Röhre mit Einzelzahn (Aufsicht auf die Ansatzfläche an der Röhrenwand). W lamellenartige Wandverstärkungen, Z Zahn. (Zeichnung: F. HeckmanN)

Abb. 5 a-e: Stephanoscyphus eumedusoides n. spec., Röhrenabschnitte aus dem gleichen Basalteil wie in Abbildung 4. a Seitenansicht des Einzelzahns, $b$ Querschnitt mit Aufsicht auf den Zahn von oben, $c-e$ Querschnitte mit den Wandverstärkungen. (Zeichnung: F. HEcKMANN)

kennbar sind und in unregelmäßiger Form, Zahl und Größe auftreten (Abb. 4, 5 c-e, vgl. Abb. 1). Wie die Querschnitte der Röhrenwandung erkennen lassen, sind sie sekundärer Natur und stellen hohle, kammerartige Wandverstärkungen dar. Meist liegen sie in der Längsrichtung der Röhre zu mehreren dicht hinter-, stellenweise auch nebeneinander. Ihre Entstehung ist vermutlich die gleiche wie bei den Zahnbildungen der Zahnkränze (Chapman \& Werner 1972); diese ist dadurch gekennzeichnet, daß sich der Weichkörper von der Röhrenwandung abhebt und daß seine Epidermis an dieser Stelle neue Röhrensubstanz ausscheidet. Im Endergebnis entsteht so ein allseitig von Periderm umgebener Hohlraum der Röhrenwandung. Die lamellenartigen Wandverstärkungen treten hauptsächlich bei älteren Tieren auf. 
Wie sich aus der Beschreibung der wesentlichen Röhrenmerkmale : Festigkeit, Farbe, Formverhältnisse, Oberflächenstruktur, Vorhandensein von Zahnbildungen und Wandlamellen im Basalteil ergeben hat, sind sie insgesamt mehr oder weniger variabel und können kaum als sichere Artunterscheidungsmerkmale bewertet werden. Für die Identifizierung von lebendfrischen oder unmittelbar nach dem Fang konservierten Tieren eignen sich am ehesten noch die dunkelbraune Färbung und robuste Beschaffenheit der Röhre sowie die Oberflächenstruktur mit den schmalen Querringen und der kaum erkennbaren Längsstreifung. Ein sicheres Merkmal ist ferner das Fehlen von Zahnkränzen, während das Vorhandensein von 1 oder 2 Einzelzähnen und von Wandverstärkungen im Basalteil wegen ihrer Variabilität nur beschränkt brauchbar ist. Als weiteres Artkennzeichen ist nach den bisherigen Fundbeobachtungen noch das Vorkommen in Höhlen anzuführen (vgl. p. 459).

\section{Der Weichkörper}

Wie bei den anderen Arten ist der Weichkörper von S. eumedusoides in seiner Morphologie von der schlanken Peridermröhre bestimmt und läßt sich in das Capitulum mit Tentakelkrone, Mundscheibe und Kragen, den langen Calix und den Basalteil aufgliedern (vgl. Chapman \& Werner 1972). Die beiden letzteren Abschnitte gehen ohne deutliche Grenze ineinander über und unterscheiden sich kaum in ihrer Anatomie, wohl aber in ihrer Funktion. Der dünnwandige Calix ist der Körperteil, in dem die wesentlichen Prozesse des aktiven Stoffwechsels ablaufen und dessen oberer Teil während der Strobilation in die Medusenanlagen umgewandelt wird. Der Basalteil des Körpers dient hauptsächlich der Speicherung von Reservestoffen; daher sind seine Wandungen verdickt. Aus diesem Abschnitt regeneriert der Polyp nach beendeter Strobilation oder nach einem zufälligen Verlust der oberen Abschnitte den vollständigen Körper.

Der Kopfreil ragt mit Tentakelkrone, eingesenkter Mundscheibe und relativ breitem Kragen (Breite ca. 0,5 mm) über die Röhrenmündung hinaus (Abb. 6). Die Zahl der Tentakel ist vom Alter bzw. von der Größe der Tiere abhängig und beträgt bei erwachsenen Tieren 40 bis 60. Die Maximalzahl war 70. Die Tentakel sind in einem Kranz angeordnet. Da sie jedoch meist abwechselnd nach oben und unten getragen werden, sind ihre Ansatzstellen parallel zur Körperachse etwas gegeneinander versetzt. Das triff vor allem für größere Tiere mit einer höheren Tentakelzahl zu. Die 4 Gastralsepten setzen an der einfachen, sich ringblendenartig erweiternden Mundöffnung an. Sie heben sich unterhalb der Mundscheibe bogenförmig von der Wand des Coelenteron $a b$, so daß ein kurzes oberes Stïck in Gastraltaschen aufgegliedert ist. Im Calix flachen sich die Septen ab und verlaufen als bandförmige, schwach ausgebildete Verdickungen nach unten in den Basalteil. Der Ringkanal (-sinus) ist im Kopfteil als horizontal verlaufender entodermaler Kanal vorhanden und öffnet sich an seiner unteren Kante durch 4 perradiale einfache Poren in den Gastralraum. Die bei anderen Arten vorhandenen kurzen Radialkanäle (WERNER 1966, 1967) sind daher bei S. eumedusoides auf einfache porenartige Offnungen reduziert, wie das auch schon für den kolonialen Polypen von Nausitboë punctata beschrieben ist (CHAPMAN \& Werner 1972). 
Die Färbung des Weichkörpers ist schwach gelblich. Nur der untere Saum des Kragens hat bei den meisten Exemplaren eine kräftiger gelbliche bis schwach bräunliche Färbung. Sie ist besonders deutlich in der wenig eingesenkten. Rinne, in der beim ausgestreckten Tier Weichkörper und Rand der Röhrenmündung verbunden sind und in der die Ausscheidung der Röhrensubstanz bei dem Längenwachstum erfolgt ( $P, A b b .1$, 6). Dieser Farbring der Kragenepidermis ist nicht bei allen Tieren gleich deutlich ent-

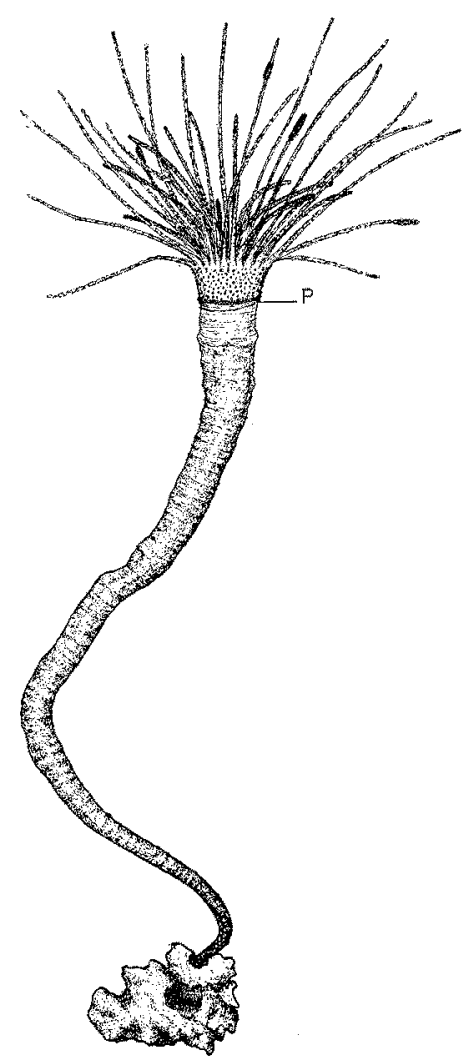

Abb. 6: Stephanoscyphus eumedusoides n. spec., Polyp mit ausgestreckter Tentakelkrone. P Pigmentring am unteren Rand des Kragens. Länge der Röhre 15,4 mm, oberer Durchmesser $1,4 \mathrm{~mm}$, unterer Durchmesser $0,2 \mathrm{~mm}$, Zahl der Tentakel 42. (Zeichnung: F. Heckmans)

wickelt, aber bei einem großen Teil doch deutlich erkennbar. Abschließend ist zu den Merkmalen des Weichkörpers zu sagen, daß er bis auf den beschriebenen Pigmentring keinerlei strukturelle Eigentümlichkeiten aufweist, die eine sichere Unterscheidung von anderen Arten ermöglichten. Das gilt auch für die Nesselzellausstattung, die später behandelt wird (pp. 456-458). Zum Alter der Polypen ist zu bemerken, daß das Einzelexemplar offenbar ein recht hohes Alter erreichen kann. Die beim Fang mindestens einjährigen Polypen wurden inzwischen 4 und 5 Jahre gezüchtet, ohne daß außer dem verlangsamten Längenwachstum andere Alterserscheinungen beobachtet wurden. Auch dies ist eine Eigenschaft, die S. eumedusoides mit den anderen Arten teilt. 


\section{Die Strobilation, die Entwicklung und Struktur der Medusoide}

Wie schon einleitend erwähnt, strobiliert $S$. eumedusoides in der für alle Scyphozoenpolypen typischen Weise durch Querteilung des Körpers, dessen obere Hälfte oder oberen zwei Drittel in die Medusenanlagen umgewandelt werden. Nach Beendigung der Strobilationsphase regeneriert der Polyp aus dem basalen Restkörper; er wächst weiter und kann später erneut strobilieren. Wenn damit im Grundvorgang der Strobilation Übereinstimmung mit den anderen metagenetischen Arten besteht, so treten in den Einzelvorgängen der Entwicklung und Morphogenese doch Unterschiede auf, die den bekannten Ablauf erheblich verändern. Die Unterschiede sind bestimmt durch die geringe Zahl, die veränderte Morphologie und Anatomie sowie das definitive Schicksal der Strobilationsprodukte und bedingen sich gegenseitig.

S. eumedusoides strobiliert nach dem polydisken Typus, wobei meist 5 bis 7 , maximal 9 Glieder in der Strobilationskette entstehen. Der kleinste in Strobilation angetroffene Polyp von 4,5 mm Röhrenlänge hatte 3 Medusenanlagen erzeugt. Mit dieser im Vergleich zu anderen Arten des Genus, bei denen in einer einzigen Strobilationsphase zahlreiche, bei einigen Species mehrere 100 bis mehrere 1000 Medusenanlagen erzeugt werden können, sehr geringen Zahl ist zunächst korreliert, daß während der Strobilation keine Streckung des Polypenkörpers eintritt. Bekanntlich streckt sich die Strobilationskette bei den Arten mit zahlreichen Medusenanlagen so erheblich, daß sie sich in der Röhre aufwinden muß, um in ihr Platz zu finden. Ferner haben die Medusenanlagen der anderen Arten von Beginn ihrer Entwicklung bis zu ihrer Ablösung die flache Scheibenform, die für die Ephyren nicht nur der Coronatae, sondern auch der Semaeostomeae und Rhizostomeae charakteristisch ist. Im Gegensatz dazu haben die Medusenanlagen von S. eumedusoides von Anfang an eine ausgeprägte Glockenform, so daß ihr Längsdurchmesser im Verhältnis zum Querdurchmesser erheblich vergrößert ist.

Das erste Anzeichen der beginnenden Strobilation besteht darin, daß der Weichkörper in seiner oberen Hälfte durch die Ansammlung von Reservestoffen in zunehmendem Maße undurchsichtig wird. Das trifft besonders für die Septen zu. Mit metamer angeordneten Verdickungen der Septalleisten werden auf diesem Anfangsstadium bereits die ersten Anlagen der Gonaden sichtbar. Zwischen diesen Verdickungen hebt sich gleichzeitig der Körper von der Röhrenwand $a b$, und es beginnt die zentripetale Einschnürung, durch die er in die Medusenanlagen aufgeteilt wird. Ihre Bildung ist ein vereinfachter Vorgang, weil sie als einfache, unvollständige Querdurchtrennung des Polypenkörpers verstanden werden kann, während die Formbildungsvorgänge bei der Strobilation der Arten mit freien Ephyren erheblich komplizierter sind. Das betriff beispielsweise die Entstehung des Gastralraumes, der bei der Entwicklung freier Ephyren neu entsteht, bei den Medusoiden von S. etumedusoides aber als Teil des Coelenteron des Polypen durch die Einschnürung direkt übernommen wird.

An den Einschnürungsstellen wächst die Körperwand als Doppellamelle ringblendenartig in zentripetaler Richtung vor, ohne dabei aber in der Mitte vollständig zu verwachsen. Die Gastralräume aller so gebildeten Medusoide bleiben daher in ständiger zentraler Längsverbindung. An den Einschnürungsstellen entsteht aus dem - auf 
die Körperachse des Polypen bezogen - unteren proximalen Blatt der Doppellamelle die Subumbrella, aus dem oberen distalen Blatt die Apikalregion der Medusoide, die hier verbunden bleiben. Ferner wächst an den Einschnürungsstellen von der Peripherie des jeweils unteren Medusoids zentrifugal und nach oben der Schirmrand aus, der sich beim weiteren Wachstum in die Randlappen und die zwischen ihnen gelegenen 8 kurzen soliden Tentakel gliedert. So entsteht die zusammenhängende Kette von Medusoiden,

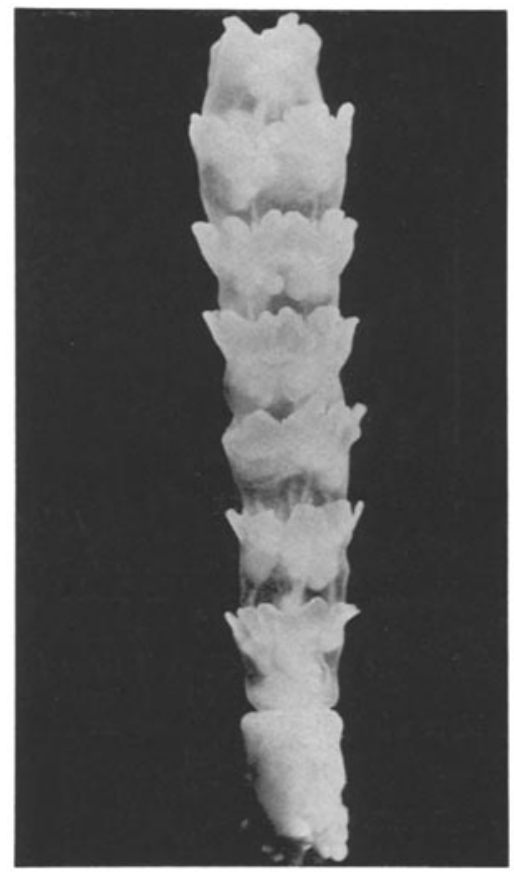

Abb. 7: Stephanoscyphus eumedusoides n. spec., Auflichtphoto einer vollständigen Strobilationskette von 7 Eumedusoiden, die mit dem oberen Teil des Polypenkörpers der Peridermröhre entnommen ist. Bei den mittleren Randlappen des von oben 3. und 4. Medusoids ist die Paarigkeit deutlich zu erkennen. Länge der Strobilationskette $9 \mathrm{~mm}$

die mit Apikalteil und Schirmrand bzw. Subumbrella teilweise verwachsen und ineinander geschachtelt sind (Abb. 7). Die Entwicklungsgeschwindigkeit ist im distalen Teil der Strobilationskette etwas größer, so daß die Differenzierung der oberen Medusoide der der unteren vorauseilt. Das unterste Medusoid, das entsprechend als jüngstes zu bezeichnen ist, bleibt mit dem Polypenkörper in fester Verbindung; hier ist die zentrale Verwachsung vollständig, so daß keine Verbindung zum Gastralraum des Polypen besteht.

Der Kopfteil des Polypen wandelt sich unter gleichzeitiger Reduktion der Tentakel in das Operculum um, das anfangs die Röhrenmündung vollständig verschließt, ohne aber nach außen einen Peridermdeckel abzuscheiden (Abb. 8). Im Verlauf der weiteren Entwicklung verkleinert sich das Operculum (Abb. 9) und wird schließlich vom obersten, distalen Medusoid vollständig resorbiert. S. eumedusoides gehört also 
zu den Arten, die in der evolutiv progressiven Reihe der Bildung bzw. Rückbildung des Opercularapparates an der Spitze stehen (vgl. WERNER 1970). Ergänzend sei erwähnt, daß der Polyp wie alle anderen bekannten Arten die Fähigkeit der Bildung eines Peridermdeckels nicht vollständig verloren hat. Sie wird jedoch nur während der seltenen

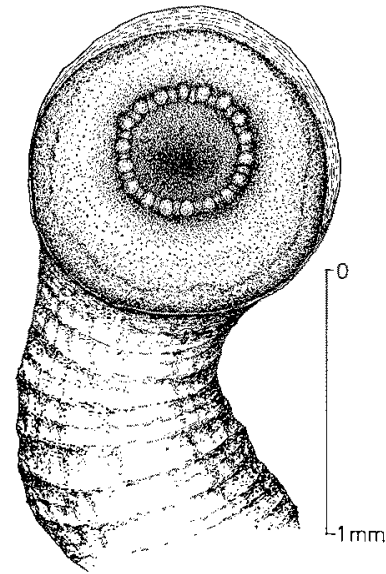

Abb. 8

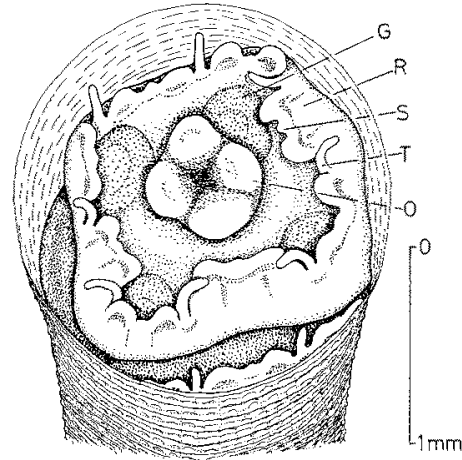

Abb. 9

Abb. 8: Stephanoscyphus eumedusoides n. spec., Oberteil einer frühen Strobila mit Aufblick auf das Operculum. Die kontrahierten Tentakel sind in Reduktion begriffen (Zeichnung: $F$. HeCKMANN)

Abb. 9: Stephanoscyphus eumedusoides n. spec., Oberteil einer fortgeschrittenen Strobila mit Aufblick auf das distale Medusoid. G durchscheinende Gonade, O Rest des Operculum, das wenig später vollständig resorbiert wird, $R$ Randlappenpaar mit Anlage des Sinnesorgans $S$, $T$ Tentakel

inaktiven Phasen realisiert, während derer sich der Polyp in die Röhre zurückzieht und den Kopfteil unter Einschlagen der Tentakel kontrahiert. Die Außenseite des Kopfteils scheidet dann eine sehr dünne Peridermscheibe $a b$, durch die die Röhre vollständig verschlossen wird.

Für die vollentwickelten Medusoide ist die bereits erwähnte Glockenform des Schirms charakteristisch (Abb. 7, 10, 12-14); die Höhe (Länge) schwankt zwischen etwa 1,0 und $1,2 \mathrm{~mm}$, die Breite (Durchmesser am Schirmrand) zwischen 1,0 und $1,5 \mathrm{~mm}$. Die Glockenform ist mit der geringen Zahl der Medusoide korreliert und ist zweifellos auch durch die Entwicklung in dem beengten Raum der Polypenröhre bedingt. Ferner ist als wesentliches Merkmal hervorzuheben, daß ihnen ein Manubrium fehlt und daß ein Mund nur in der Form der Offnung an der zentralen Verbindung je zweier Medusoide erhalten ist. Nur das distale Medusoid besitzt in den späteren Stadien nach der vollständigen Resorption des Operculum eine Mundöffnung. Sie besteht aus einer einfachen vier- oder rechteckigen Offnung ohne kontraktile Mundlippen. Ein weiteres auffälliges Merkmal ist das Fehlen der für alle Coronaten-Medusen so charakteristischen Ringfurche zwischen dem Zentralteil des Schirms und den peripheren Randbezirken seiner Exumbrellarseite. Nur wenn sich die Strobilationskette bei einer Reizung von außen kontrahiert, wird eine Ringfurche wenigstens andeutungsweise er- 
kennbar, die aber nicht als stabile Struktur existiert. Auch dieses Merkmal ist auf die allgemein veränderte und vereinfachte Morphologie der Medusoide zurückzuführen, ist also sekundärer Natur und kann nicht als Primitivmerkmal gedeutet werden.

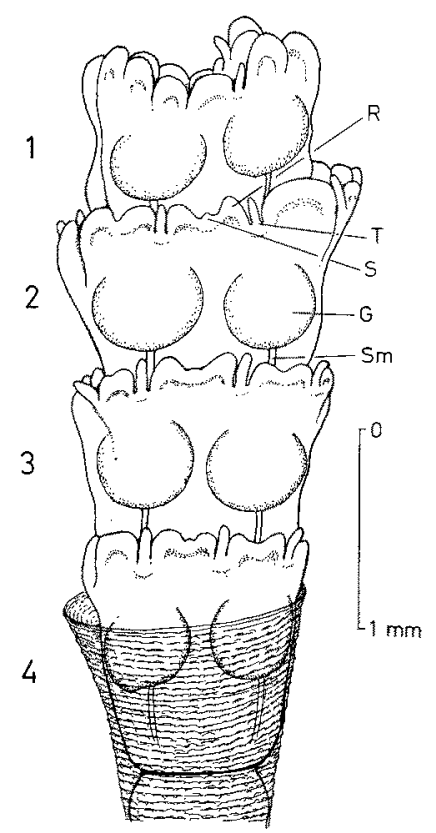

Abb. 10

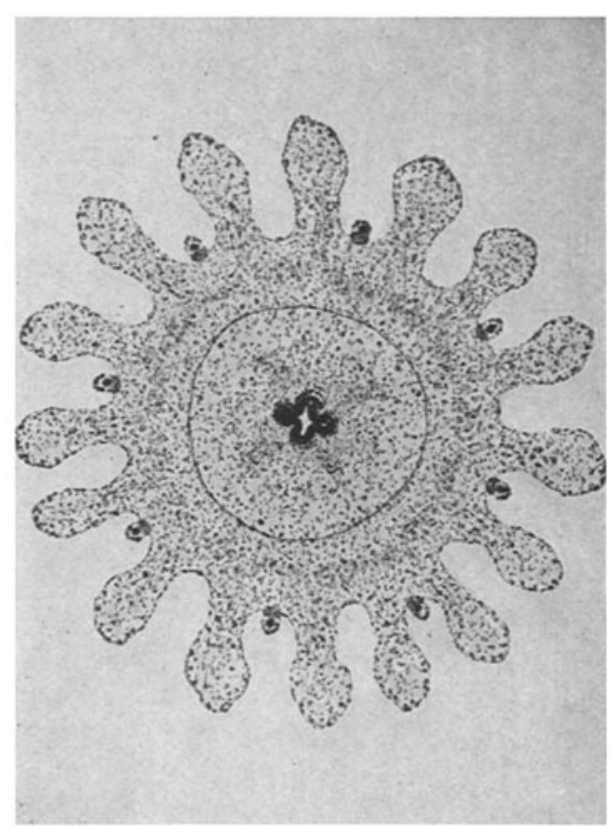

Abb, 11

Abb. 10: Stephanoscypbus eumedusoides n. spec., Strobilationskette von 4 Eumedusoiden, die vorzeitig aus der Polypenröhre ausgetreten ist. Zu beachten sind die Schirmrandstrukturen: bei Medusoid 1 ist die Beschaffenheit der Randlappen durch die Kontraktion des Schirmrandes nicht mehr eindeutig erkennbar; bei den Medusoiden 2-4 ist jeweils der mittlere Randlappen paarig und weist auch die Anlage des Sinnesorgans auf. Die übrigen Randlappen sind paarig oder unpaar ohne Anlage des Sinnesorgans. G Gonade, R Randlappenpaar, S Anlage des Sinnesorgans, Sm Septalmuskel, T Tentakel

Abb. 11: Nausithoë punctata (KöLliker 1853), soeben abgelöste Ephyra. Vergleiche die Schirmrandstrukturen mit denen der Eumedusoide von S. eumedusoides in den Abbildungen 9 und 10. Durchmesser der Ephyra 2,5 mm

Wie schon einleitend erwähnt, gelten entsprechende Verhältnisse auch für die Entwicklung der Randorgane des Schirms, deren Einzelvorgänge wegen der geringen Durchsichtigkeit der Röhrenwandung normalerweise von außen nur schwer verfolgt werden können. Diè Morphologie der Medusoide wurde daher zunächst an der gealterten Kette untersucht, die vom Polypen bereits aus der Röhre herausgeschoben war (Abb. 13). In diesem Stadium kann der Schirm der Medusoide aber bereits geschrumpft sein, was auch für die Organe des Schirmrandes zutrifft. Seine oktomere Gliederung ist zwar durch die Achtzahl der Tentakel und der zwischen ihnen liegenden Randlappen meist noch erhalten; doch war die Struktur der letzteren oftmals nicht mehr eindeutig erkennbar. Das war besonders häufig bei dem distalen Medusoid der Fall, bei dem die 
Schrumpfung des Schirms dazu führen konnte, daß seine Randorgane zu 4 Gruppen zusammengezogen waren und daß so eine tetramere Gliederung des Schirms vorgetäuscht wurde.

Erst die genauere Untersuchung der Ausbildung des Schirmrandes jüngerer Medusoide an Strobilationsketten, die in günstigen Einzelfällen teilweise aus der Röhre herausragten (Abb. 10) oder beim Einblick in die Röhrenmündung von oben betrachtet werden konnten (Abb. 9), ferner vor allem die Prüfung ganzer Strobilationsketten, die experimentell auf verschiedenen Stadien von der Röhre befreit waren (Abb. 7), ergab mit aller Deutlichkeit, daß die Entwicklung des Schirmrandes zu der prinzipiell gleichen Gliederung in 8 Randlappenpaare führen kann, die für die Ephyren des Genus Nausithoë zutrifft. Allerdings bleibt die Entwicklung auf einem frühen Stadium stehen, so daß die Randlappen der Medusoide eine kurze, breite Form annehmen und daß auch der Spalt zwischen den Lappen eines Paares verbreitert ist. Ebenso bleibt die Anlage des Sinnesorgans, die am Grunde des Spaltes zwischen den Lappen eines zusammengehörigen Paares als rundliche Vorwölbung erkennbar ist, auf dem Anfangsstadium stehen, und die weitere Differenzierung zur bläschenförmigen Statozyste mit Sinneszellen und Statolithen unterbleibt. Zum Vergleich ist in Abbildung 11 die Ephyra einer Art mit freien Medusen, nämlich von Nausitboë punctata, dargestellt; sie macht die prinzipiell übereinstimmende Gliederung des Schirmandes deutlich. Der Ephyra fehlen lediglich noch die Tentakel, die erst während ihrer pelagischen Phase auswachsen.

Andererseits hat aber die direkte Beobachtung immer wieder ergeben, daß die Ausbildung des Schirmrandes auch bei den jungen, noch in Entwicklung begriffenen Medusoiden variieren kann, weil bei ihnen neben paarigen auch unpaare Randlappen auftreten können, wobei dann auch die Anlage des Sinnesorgans fehlt (Abb. 10). Die Einzelmedusoide einer Strobilationskette können sich in der Zahl der paarigen oder unpaaren Randlappen verschieden verhalten, ohne daßs eine feste Regel erkennbar ist. In seltenen Extremfällen haben alle Medusoide nur paarige oder unpaare Randlappen; doch sind die Zwischenstufen mit wechselnder Zahl des einen oder anderen Typs häufiger. Zu erwähnen ist noch, daß die 8 kurzen, soliden Tentakel frühzeitig aus knopfförmigen Anlagen hervorgehen. Die Entodermzellen im Inneren liegen chordioid hintereinander, wie das für alle Coronatenmedusen zutrift.

Nach weiteren neuen Beobachtungen an strobilierenden Polypen, die im Juni 1974 in Marseille unmittelbar nach dem Fang untersucht werden konnten, ist es nicht auszuschließen, daß die beschriebene Variabilität in der Ausbildung der Schirmrandorgane von Außenbedingungen abhängig ist. Bei den Marseiller Polypen wurde nämlich die Reduktion des Schirmrandes zu 8 unpaaren Randlappen ohne jede Andeutung der Anlage der Sinnesorgane in so ausgeprägter Weise beobachtet, wie sie bei den Kulturexemplaren in gleich deutlicher Form niemals angetroffen wurde. Der vermutete Einfluß der unterschiedlichen Lebensbedingungen bedarf allerdings noch der experimentellen Bestätigung.

Als Ergebnis aller Beobachtungen ist festzuhalten, daß die Organe des Schirmrandes der Medusoide von $S$, eumedusoides der Anlage nach die gleiche Gliederung aufweisen, wie sie in voll differenzierter Form bei den Ephyren der Arten mit freischwimmenden Medusen ausgeprägt ist. Nach der oktomeren Gliederung des Schirms ist der verwandtschaftliche Zusammenhang mit Arten, deren Medusen dem Genus Nausitbö̈ 
angehören, nicht zweifelhaft. Ebenso läßt das Vorhandensein der Tentakel und der eben noch erkennbaren Anlagen der Sinnesorgane es als unzweifelhaft erscheinen, daß $S$. eumedusoides von Vorfahren abstammt, die freischwimmende Medusen gehabt haben. Die abgeänderte Entwicklung der Strobilationsprodukte zu sessilen Medusoiden muß nach allem als Ergebnis einer progressiven Evolution betrachtet werden, die zu

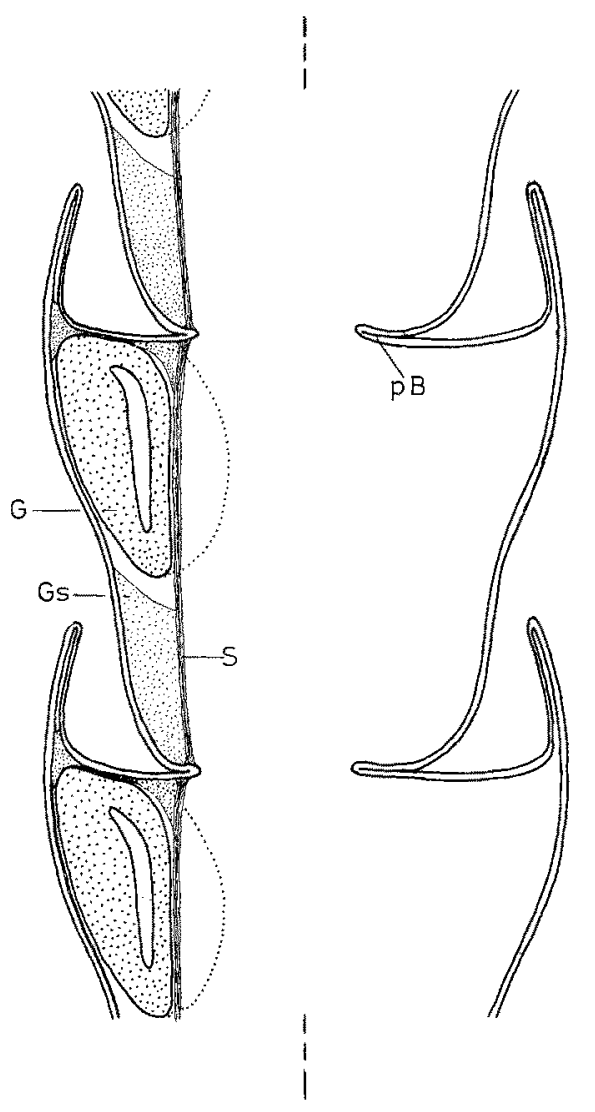

Abb. 12: Stephanoscyphus eumedusoides n. spec., schematischer Längsschnitt durch den Mittelteil einer Strobilationskette. Der Schnitt ist links von der Mittelachse (gestrichelt) durch einen Interradius, rechts durch einen Perradius gelegt. G Gonade, deren volle Größe durch die punktierte Linie angedeutet ist (Querschnitt nierenförmig), Gs Gastralseptum, pB präformierte Bruchstelle, S Septalmuskel

sekundären Vereinfachungen geführt hat. KüHN (1913) ist bei seinen Untersuchungen über die Ausbildung der sessilen Gonophoren der Hydroidpolypen zu dem gleichen Ergebnis gekommen; er hat in überzeugender Weise begründet, daß die unterschiedlichen Reduktionserscheinungen als Stufen einer progressiven Evolution gedeutet werden müssen.

Reduktionserscheinungen kennzeichnen weiterhin die strukturelle Differenzierung der Medusoide (Abb. 12). Wenn auch die eingehende histologische Analyse noch aus- 
steht und einer späteren Arbeit vorbehalten bleiben muß, so lassen die vorläufigen Untersuchungen doch bereits einige Merkmale der Vereinfachung erkennen. So fällt sofort auf, daß der Schirm dünnwandig bleibt, weil die für die metagenetischen Formen so charakteristische Schirmgallerte fehlt. Damit hängt es auch zusammen, daß die Schirmwandung trotz ihrer geringen Dicke anfangs wenig transparent ist, was sich erst auf den späteren Stadien ändert. Ferner ist die Entodermschicht des Gastralraumes aus niedrigem Plattenepithel aufgebaut, das keine Verdauungsfunktionen mehr hat. Entsprechend fehlen auch Gastralfilamente. Ebenso ist die Muskulatur des Schirms reduziert, da ihm der ringförmige Coronarmuskel fehlt. Daher haben die Medusoide nicht die Fähigkeit zu pulsierenden Schirmkontraktionen. Das Muskelsystem ist jedoch nicht vollständig rückgebildet, wie unmittelbar aus der Fähigkeit der Strobilationskette erschlossen werden kann, sich bei Reizungen zu kontrahieren und in die Röhre weiter zurückzuziehen. Diese Kontraktionsfähigkeit ist auch in den Strobilationsketten vorhanden, die experimentell aus der Röhre herausgenommen sind. Sie ist auf die Ausbildung von 4 dünnen Längsmuskelsträngen zurückzuführen, die die Gastralräume in den Interradien durchziehen und offenbar aus den Muskelsträngen der Polypensepten direkt hervorgehen. Die Muskelstränge der Medusoide liegen anfangs am freien Rand zarter Gastralsepten, die an der inneren Schirmwand ansetzen, aber später reduziert werden. Im Endzustand verlaufen die Längsmuskelstränge frei im Gastralraum jedes Medusoids und sind jeweils nur interradial an den Verbindungsstellen je zweier Medusoide befestigt.

Auf die spärliche Ausstattung der Medusoide mit Nesselzellen, die praktisch funktionslos bleiben, wird später noch eingegangen (pp. 456-458). Ferner ist erwähnenswert, daß die Epidermis der Medusoide schlagende Geißeln besitzt; das ist deswegen hervorzuheben, weil es sich um eine Meduseneigenschaft handelt. Der Polyp besitzt epidermale Geißeln nur am Capitulum, das heißt am Kragen, an den Tentakeln und an der Mundscheibe, nicht aber an der von der Röhre umschlossenen Körperwand. Sie müssen also bei der Bildung der Medusoide (und der Ephyren metagenetischer Arten) neu entstehen.

Das Gastralsystem der Medusoide ist stark vereinfacht und auf einen einheitlichen Raum reduziert, der anfangs durch die Septen eine Gliederung in 4 Gastraltaschen aufweist. Bei den metagenetischen Arten besteht das Gastralsystem bekanntlich aus dem Zentralmagen und dem peripheren Kranzdarm, der sich in die taschenförmigen Aufzweigungen der Randlappen erweitert. Ein Überrest der ursprünglichen Form kann darin erblickt werden, daß sich der Gastralraum der Medusoide in die Basis der kurzen Randlappen fortsetzt.

Die bei den Medusen von Nausithoë in Achtzahl vorhandenen Gonaden sind auf 4 reduziert. Bei den jüngsten Entwicklungsstadien der Medusoide läßt sich beobachten, daß sie paarig angelegt werden, da sie zuerst als rundliche Verdickungen auf jeder Seite eines Gastralseptums in Erscheinung treten. Sie vergrößern sich bei der weiteren Entwicklung erheblich und nehmen im vollentwickelten Zustand vor dem Ausstoßen der Keimzellen einen großen Teil des Gastralraums ein. Manchmal bleibt die anfängliche Nierenform äußerlich sichtbar, die ebenfalls auf die paarige Anlage hinweist. Oft haben die Gonaden auch (von außen gesehen) eine rundliche oder ovale Form. Nach 
dem Ausstoßen der Keimzellen sind die Gonaden geschrumptt und weisen dann durchweg einen nierenförmigen Querschnitt auf.

Bei $S$. eumedusoides ist also sekundär der als ursprünglich zu betrachtende $\mathrm{Zu}$ stand der Vierzahl der Gonaden wiederhergestellt, wie er nach der Vierzahl der Septen für die Vorfahren der rezenten Scyphozoen postuliert werden muß. Als Stammform der Scyphozoen und aller Cnidarier wird bekanntlich ein sessiler, tetramer gebauter Polyp angesehen, der sich sexuell fortpflanzte und bei dem die Keimzellen in den Gastralsepten gebildet wurden.

Die Gonaden haben eine anfangs gelbliche Farbe. Ihre fortschreitende Reifung läßt sich außer an der Vergrößerung auch an der Einlagerung eines bräunlichen Pigmentes erkennen. Zuweilen gelangen nicht sämtliche 4 Gonaden zur vollen Entwicklung und Bildung von Keimzellen, so daß ein Medusoid nur 3 oder 2 Gonaden oder sogar nur 1 Gonade besitzt. Dabei bleiben die Primäranlagen der nicht zur Entwicklung gelangten Gonaden als interradiale Verdickungen sichtbar. Die Ursache ist vermutlich darin zu suchen, daß der Polyp nicht genügend Reservestoffe angesammelt hatte.

Die Medusoide einer Strobilationskette weisen alle möglichen Formen von Hermaphroditismus auf, wie schon früher mitgeteilt wurde. So können Medusoide rein männlichen und rein weiblichen Geschlechts auftreten, ferner kann das gleiche Medusoid männliche und weibliche Gonaden besitzen, und in der gleichen Gonade können schließlich Eier und Spermien erzeugt werden. Irgendeine Regel, etwa in der Sexualität der distalen älteren und proximalen jüngeren Medusoide, war bisher nicht zu erkennen.

Es wurde auch bereits erwähnt, daß der regenerierende Polyp die Kette der Medusoide aktiv aus der Röhre herausschiebt und schließlich abstößt (Abb. 13). Er ist also in der Lage, mit Hilfe der Reservestoffe des basalen Restkörpers den oberen Körperteil neu aufzubauen. Die Tentakelkrone wird bereits in der Röhre ausgebildet, so daß der Polyp nach dem völligen Ausstrecken und Abstoßen der Medusoidkette sofort wieder mit der Nahrungsaufnahme beginnen kann. Die Dauer der anschließenden Phase der Erholung und des weiteren Wachstums bis zum Eintritt der nächsten Strobilation hängt von der Ernährung und der Temperatur ab; bei konstanten Temperaturen von 15 bis $17^{\circ} \mathrm{C}$ und regelmäßiger Nahrungszufuhr betrug sie 4 bis 6 Monate.

\section{Die Entwicklung der Planula bis zum jungen Polypen}

Wie für alle Scyphozoen typisch, werden die Keimzellen durch Platzen der Gonadenwand frei und gelangen so in den Gastralraum des betreffenden Medusoids, wo die Furchung und Entwicklung zur begeißelten Planula erfolgt, wo also auch die Besamung stattfinden muß. Es ist anzunehmen, daß bei den Strobilae benachbarter Polypen Fremdbesamung die Regel ist. Sie ist dadurch möglich, daß die Spermien aus der Mundöffnung des distalen Medusoids austreten und auf dem gleichen Wege in die Gastralräume der von anderen Polypen erzeugten Medusoide eindringen können. Andererseits wurde durch zahlreiche Isolierungsversuche nachgewiesen, daß Selbstbefruchtung in der gleichen Kette möglich ist. Die Voraussetzung dafür ist damit gegeben, daß wie erwähnt die Gastralräume sämtlicher Medusoide einer Strobilationskette in ständiger zentraler Verbindung bleiben, so daß die Spermien einer Gonade sämtliche Eizellen 
erreichen können. Daß bei den Isolierungsversuchen die Zahl der erzeugten Planulae stark verringert ist, kann als Hinweis dafür gelten, daß Fremdbesamung als Normalfall zu gelten hat.

Die Eizellen haben eine weißgelbliche Färbung, die sich während der Furchung und weiteren Entwicklung häufig in rötlich-violett ändert. Die vollentwickelten Planulae können durch die Mundöffnung des distalen Medusoids ins freie Wasser austre-

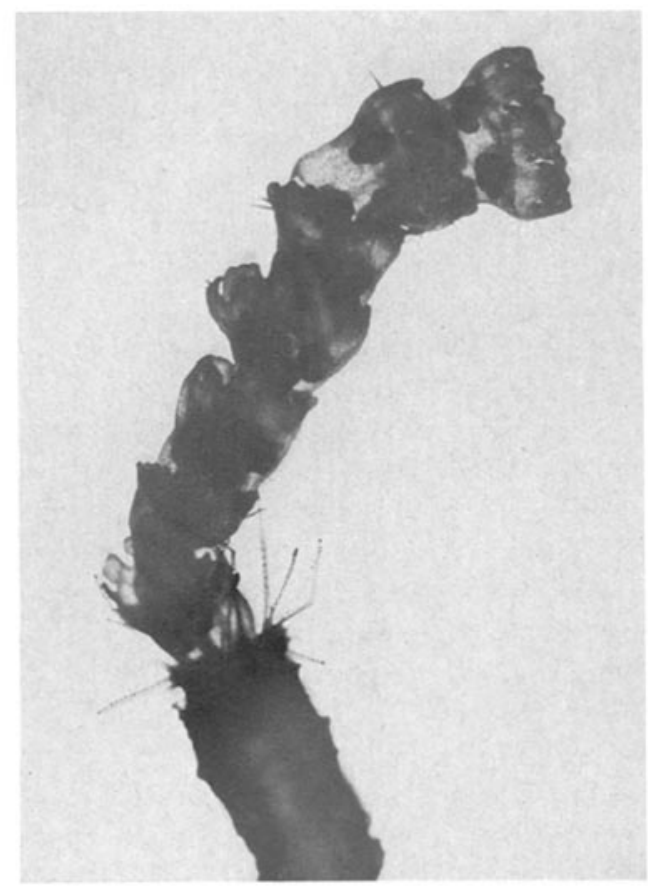

Abb. 13

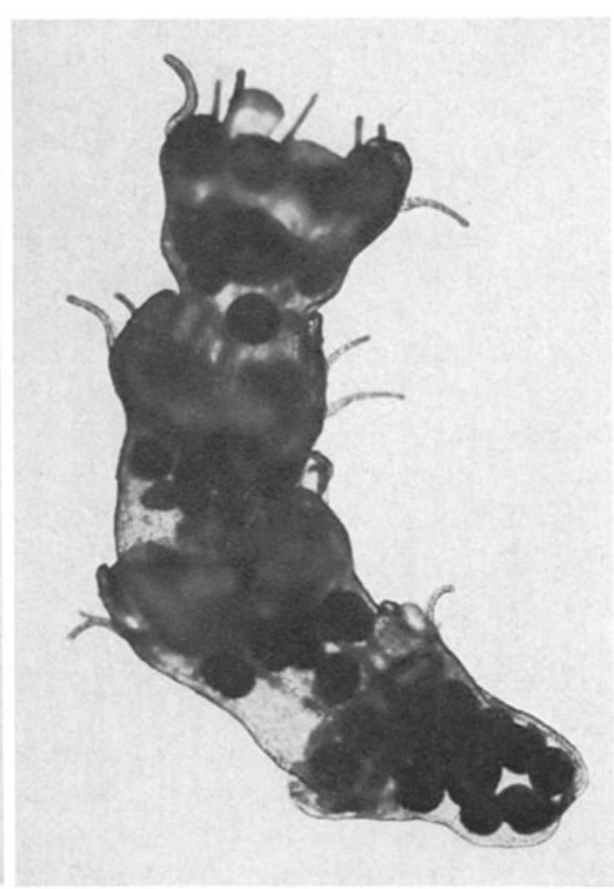

Abb. 14

Abb. 13: Stephanoscypbus exmedusoides n. spec., oberer Teil eines Polypen mit einer Kette von 7 Eumedusoiden, die am Ende der Strobilationsphase aus der Röhre herausgeschoben ist. Der Polyp hat bereits die Tentakelkrone regeneriert, ist aber noch mit der Kette verbunden. (Aus WERNER 1971a)

Abb. 14: Stephanoscypbus eumedusoides n. spec., Teil einer Strobilationskette, die vom Polypen abgestoßen ist und vor dem Zerfall steht. Im Apikalteil der Medusoide, vor allem im untersten Medusoid, zahlreiche schlüpfreife Planulae

ten. Das läßt sich auch ohne direkte Beobachtung daraus erschließen, daß in den Kulturschalen Planulae gefunden werden, auch wenn die Medusoidkette noch vollständig in der Polypenröhre eingeschlossen ist. Der größte Teil der Planulae, die sich vielfach in den unteren proximalen Medusoiden konzentrieren, wird jedoch erst frei, wenn die vom Polypen ausgestoßene Kette zerfällt und sich in die Einzelmedusoide auflöst (Abb. 14). Dabei erfolgt die Trennung zuerst in den Perradien der apikal-umbrellaren Verbindungsstellen der Medusoide, so daß hier 4 horizontale Schlitze entstehen und die Lage präformierter Bruchstellen anzeigen (Abb. 12). Anschließend kommt es dann auch 
zur interradialen Trennung. Nicht selten bleiben mehrere Medusoide der ausgestoßenen Kette bis zuletzt verbunden und zerfallen gemeinsam.

Die Dauer der Entwicklung vom Strobilationsbeginn bis zum Freiwerden der Planulae hängt ebenfalls von der Temperatur ab; sie beträgt bei 15 bis $17^{\circ} \mathrm{C} 6$ bis 11 , meist 9 bis 11 Wochen. Die Zahl der in einer Strobilationsphase erzeugten Planulae ist ziemlich variabel und wechselt mit der Größe des Polypen, der Zahl der Medusoide und offenbar auch mit der Menge der verfügbaren Reservestoffe. So wurden Zahlen von 20 bis zu maximal etwa 150 Planulae pro Medusoidkette ermittelt. Das einzelne Medusoid kann danach einige wenige bis zu etwa 20 bis 30 Planulae erzeugen. Die Entwicklungsgeschichte von $S$. eumedusoides ist nach allem durch sessile Gonophoren und die Larviparie gekennzeichnet.

Die Kultur metagenetischer Scyphozoen über den vollen Lebenszyklus ist stets mit Schwierigkeiten verbunden, wobei die Aufzucht der Medusen bis zur Geschlechtsreife die langwierige und kritische Phase darstellt. Bei S. eumedusoides entfällt diese Erschwerung, weil die pelagische Phase der Medusengeneration vollständig eliminiert ist. Der Hermaphroditismus, die Möglichkeit der Selbstbesamung und die Larviparie sind weitere entwicklungsbedingte Vorteile. Für die Kultur der Art über den vollen Lebenszyklus ist es also nur notwendig, die Planulae über die planktische Phase hinweg bis zur Anheftung und Umwandlung in den Jungpolypen zu bringen. Entsprechende Versuche verliefen erfolgreich.

Die soeben ausgeschlüpfte Planula hat die übliche länglich-ovoide Form mit verdicktem Vorderende. Ihre Länge beträgt 0,3 bis $0,35 \mathrm{~mm}$, maximal $0,4 \mathrm{~mm}$, der Durchmesser des Vorderendes $0,2 \mathrm{~mm}$. Der primäre Gastralraum ist bereits vorhanden und nimmt etwa die beiden vorderen Drittel ein. Im Ektoderm der Planula sind funktionsfähige Nesselzellen ausgebildet, die dem Typ der heterotrichen mikrobasischen Eurytelen angehören. Bei den älteren Planulae kommt dann auch der zweite Typ der holotrichen Haplonemen zur Entwicklung. Auf diesem Stadium sind die Nesselzellen auf das hinsichtlich der Bewegung hintere Ende konzentriert, das dem Oralpol entspricht. Unter gleichzeitiger Rechtsdrehung um die Körperachse (das heißt von vorn gesehen im Uhrzeigersinn) bewegen sich die Planulae schwimmend in weiten Rechtsspiralen. Sie besitzen die ökologisch offenbar wichtige Fähigkeit, bei Störungen, etwa bei heftiger Wasserbewegung oder auch bei unmittelbarem Kontakt den Geißelschlag einzustellen und sich zu Boden sinken zu lassen. Während der Anfangszeit der planktischen Phase schwimmen sie meist in Oberflächennähe, um dann später den Boden und ein geeignetes Substrat aufzusuchen.

In den Kulturen erfolgte die Anheftung am Boden der Glasschalen oder an dargebotenem Ansatzmaterial, etwa an kleinen Stücken von Peridermröhren der gleichen Art. Die Dauer der plankrischen Phase betrug bei 15 bis $17^{\circ} \mathrm{C}$ meist 3 bis 4 Wochen; doch hefteten sich die Planulae in Einzelfällen bereits nach 1 bis 2 Wochen an. Dies geschieht in der bekannten Weise mit dem Bewegungsvorderende, dem aboralen Pol, der sich in die Haftscheibe umwandelt. Dabei verbreitert sich der ganze Keim und flacht sich ab. Der obere Pol des angehefteten Keims erfährt zunächst eine schwache Einsenkung, in deren Mitte anschließend der Mund durchbricht. In den Perradien entstehen kurz danach die Anlagen der 4 Primärtentakel, die stets gleichzeitig in Erscheinung treten. Die Lagebeziehung ist deswegen eindeutig erkennbar, weil annähernd zur glei- 
chen Zeit auch die interradial gelegenen. Septen gebildet werden. Wenig später erhebt sich in der Nähe der Peripherie schornsteinförmig die erste Anlage der runden Peridermröhre, die an der vom Ektoderm des Umwandlungskeims ausgeschiedenen feinen Peridermhïlle des Basalteils ansetzt (Abb. 15a, b).

Auf dem Stadium mit 4 Primärtentakeln (Abb. 15b) beginnen die jungen Polypen mit der Nahrungsaufnahme. Dabei läßt sich beobachten, daß sich die Septen anfangs in den Basalteil hinein erstrecken, der die Form einer flachen Kuppel hat und in den

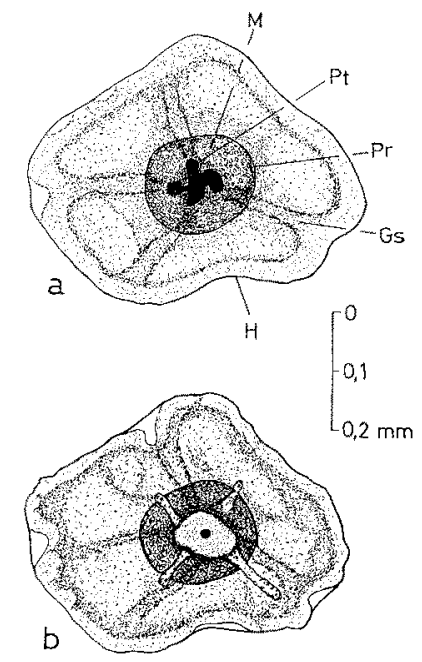

Abb. 15 a, $b$ : Stephanoscyphus eumedusoides n. spec., Entwicklung des Jungpolypen a 8 Tage, $b 13$ Tage nach der Anheftung der Planula, Aufsicht von oben. Gs Anlage eines Gastralseptums, $\mathrm{H}$ Haftscheibe, $M$ Mundscheibe mit Mundöffnung, Pr Peridermröhre, Pt Primärtentakel. (Zeichnung: F. HeCKMANN)

sich der ganze Weichkörper zurückziehen kann. Auch der primäre Gastralraum liegt anfangs in diesem Basalteil, in den die Nahrungsbestandteile aufgenommen werden. Beim weiteren Wachstum, das durch die Verlängerung der Röhre nach oben gekennzeichnet ist, erfolgt dann die Sonderung in die basale Haftscheibe, den basalen Stiel, den Calix und das Capitulum.

\section{Nesselzellausstattung}

Wie bei allen bisher untersuchten Polypen und Medusen der Coronatae (vgl. WERNER 1965) besteht auch bei S.eumedusoides die Nesselzellausstattung, das Cnidom (WEILL 1934), aus den beiden Kategorien der holotrichen Haplonemen und heterotrichen mikrobasischen Eurytelen. Ihre Verteilung bei dem Polypen, dem Medusoid und der Planula sowie die Größenverhältnisse sind in der Tabelle 2 zusammengestellt. Für den Polypen ist hervorzuheben, daß der Endteil der Tentakel fast ausschließlich mit Haplonemen besetzt ist, die in unvollständigen Ringen oder Spiralen angeordnet sind, 
Stephanoscyphus eumedusoides n. spec.

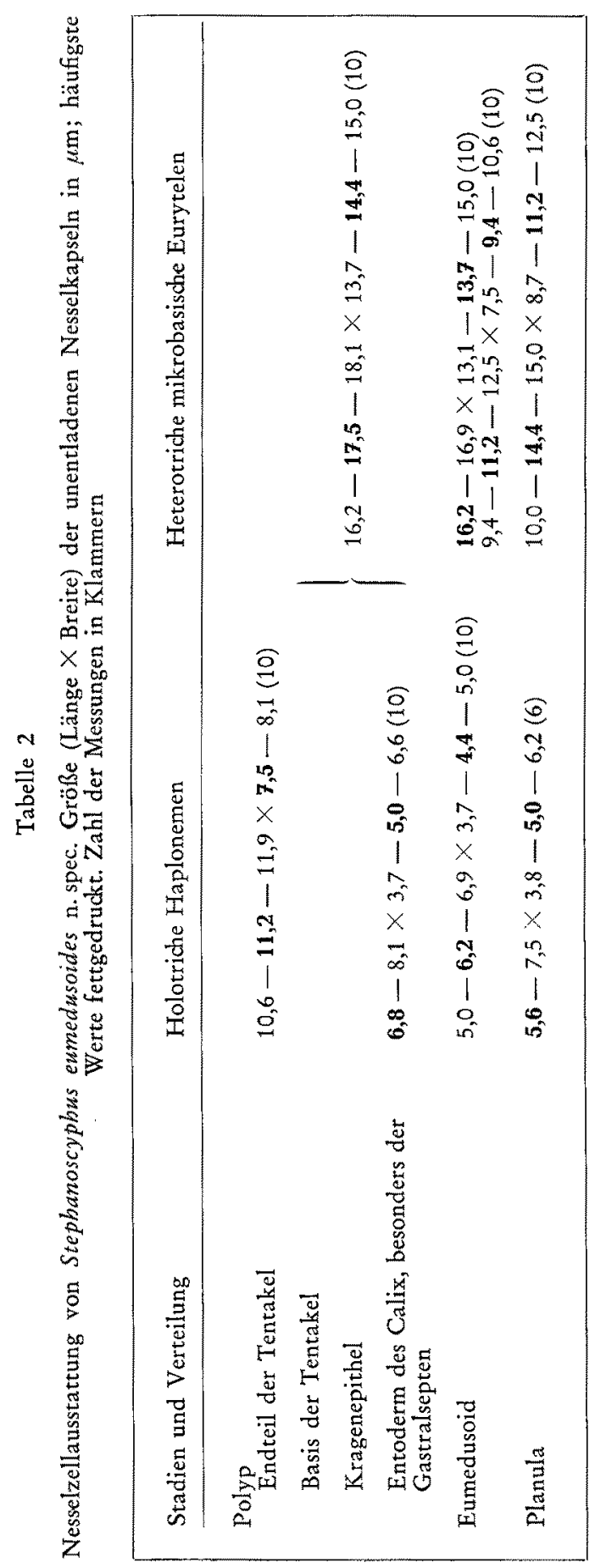


während auf der oralen Seite der Tentakelbasen die großen Eurytelen angehäut sind. Dieser Typ findet sich überwiegend auch in der Epidermis des Kragens, so daß sie bei der Betrachtung mit schwächerer Vergrößerung einen ausgesprochen "grobkörnigen" Eindruck macht.

Für die Eumedusoide, die ständig von der Polypenröhre umgeben sind und keine Nahrung aufnehmen, ist der Besitz von Nesselzellen bedeutungslos geworden. Sie sind jedoch nicht vollständig reduziert, da sich funktionsfähige Nesselkapseln in geringer Zahl an den Tentakeln und etwas häufiger in einem schmalen Saum auf der exumbrellaren Seite der Randlappen finden. Sie gehören überwiegend dem Typ der Eurytelen an, der in 2 Größenklassen vertreten ist.

\section{Verbreitung und Ókologie}

Als sedentärer Organismus benötigt Stephanoscyphus für die Anheftung der planktischen Planula, aus der sich der Polyp entwickelt, ein geeignetes sauberes und festes Substrat, z. B. Felswände von Steilküsten oder in submarinen Höhlen sowie felsige Meeresböden. Ebenso können ihm die Skelette von Korallen, die Kalkröhren von sedentären Polychaeten und Gastropoden, die Schalen von Muscheln und Schnecken als Substrat dienen, die an Felsen angeheftet sind. Ferner bieten Sedimentböden (Kies, Grob-, Fein- und Schlicksand) Existenzmöglichkeiten, wenn auf ihrer Oberfläche gröBere oder kleinere Festkörper wie Steine, Schlacken, Korallenbruchstücke, lebende Muscheln und Schnecken sowie deren Schalen vorhanden sind. In tropischen Meeren ist der Schelfabhang unterhalb von Korallenriffen, dessen obere Bodenschicht zum großen Teil aus Korallenbruchstücken, Schnecken- und Muschelschalen besteht, erfahrungsgemäß ein Biotop, in dem Stephanoscyphus regelmäßig vorkommt.

Hinsichtlich der Vertikalverbreitung ist zu beobachten, daß die Arten dieses Genus nicht im Eulitoral, sondern im mittleren bis unteren Sublitoral leben und im anschließenden Bathyal, Abyssal und Hadal bis zu $7000 \mathrm{~m}$ Tiefe gefunden worden sind (KRAMP 1959). Eine Ausnahme machen nur die japanische koloniebildende Art S. racemosus, die in Tiefen von 5 bis $10 \mathrm{~m}$ vorkommt, also im obersten Sublitoral, sowie die beiden Marseiller Höhlenformen S. eumedusoides und S. planulophorus. Die letztere Art muß nach den wenigen Funden vorläufig noch als selten betrachtet werden; sie wurde zusammen mit S. eumedusoides gefunden (WERNER 1971b). Offenbar hat sie die gleiche Vertikalverteilung wie diese Art, die bislang in Tiefen von 2-3 m bis maximal $43 \mathrm{~m}$ gesammelt wurde (Tabelle 1). Die Vertikalverbreitung von S. eumedusoides reicht also vom obersten bis mittleren Sublitoral. Allerdings läßt sich die untere Grenze bisher noch nicht genau festlegen; es ist nicht auszuschließen, daß die Art auch in tiefer gelegenen submarinen Höhlen vorkommt, die für den Taucher nicht mehr erreichbar sind.

Auch die Angaben über die Horizontalverbreitung müssen als vorläufig gelten. Sicher ist, daß S. eumedusoides in den submarinen Höhlen der Mittelmeerküste bei Marseille nicht selten, stellenweise sogar häufig ist. Die anderen Fundangaben nach konserviertem Material sind zufälliger Natur. Immerhin haben sie gezeigt, daß die Verbreitung im westlichen Mittelmeer von der Nordküste über den Golf von Neapel 
(Sorrent) bis an die afrikanische Küste (Tunis) reicht und daß die Art auch an der Atlantikküste (Portugal) vorkommt. Es ist sehr wahrscheinlich, daß weitere Fundorte bei einer gezielten Suche entdeckt würden. Die Suche müßte auch auf das östliche Mittelmeet ausgedehnt werden, aus dem (außer von der Adriaküste) bislang keine einzige Fundmeldung für eine Stephanoscyphus-Art vorliegt.

Wesentlich ist, daß S. eumedusoides mit zwei Ausnahmen bisher nur in submarinen Höhlen gefunden wurde. Die beiden Ausnahmen betreffen wenige Exemplare, die $\mathrm{zwar}$ im freien Meer, in einem Fall aber unter Steinen, im anderen in inneren Hohlräumen eines größeren, zerklüfteten Steines gefunden wurden, in Kleinbiotopen also, die hinsichtlich ihrer Eigenschaften dem Höhlenmilieu durchaus vergleichbar sind. Alle bisherigen Beobachtungen und Funde sprechen dafür, daß der Polyp als Höhlenform angesehen werden muß.

Eine Bestätigung dafür hat sich im Frühjahr 1974 ergeben, als im Golf von Neapel und Marseille bei der gezielten und erfolgreichen Suche nach anderen solitären Stephanoscyphus-Arten Dredgefänge auf geeigneten Sedimentböden in Tiefen von 50 bis $85 \mathrm{~m}$ (Neapel) und 70 bis $300 \mathrm{~m}$ (Marseille) gemacht wurden. Dabei wurde nicht ein einziges Exemplar von S. eumedusoides gefunden, obwohl das stellenweise häufige Vorkommen einer anderen, noch unbekannten Art angezeigt hatte, daß die untersuchten Stellen geeignete Existenzmöglichkeiten bieten. Das ist für die Region von Marseille deswegen hervorzuheben, weil die befischten Sedimentböden in geringer Entfernung der Inseln liegen, in deren submarinen Höhlen $S$. eumedusoides häufig ist.

Welche Eigenschaften des Höhlenmilieus : Lichtmangel, geringere Wasserbewegung und in Zusammenhang damit wohl auch verringertes Nahrungsangebot, weniger scharf ausgeprägte Temperaturgradienten - um nur die wichtigsten zu nennen (vgl. Rredr 1966) - dafür verantwortlich zu machen sind, daß S. eumedusoides nur hier vorkommt, läßt sich vorerst nicht entscheiden. Der Nahrungsfaktor kann ausgeschlossen werden, da ja die Planula das Entwicklungsstadium ist, dessen Reaktionen für das Zustandekommen der lokalen Besiedelung entscheidend sind. Die Wimperlarve nimmt keine (wenigstens keine partikuläre) Nahrung auf. Andererseits spielt der Nahrungsfaktor insofern eine Rolle, als die Oberflächenstruktur der Polypenröhre mit dicht aufeinander folgenden, schmalen Querringen ein Indiz für langsames Wachstum ist, so daß man daraus auf ein verringertes Nahrungsangebot in den Höhlen schließen darf, in denen der Polyp angetroffen wird.

Die Reaktion der Planula auf Wasserbewegung könnte ein Hinweis auf eine mögliche Ursache sein, nämlich auf die Bevorzugung von Zonen schwacher Wasserbewegung. Doch bedarf diese Vermutung noch der Prüfung durch entsprechende Experimente unter Einbeziehung der Planulae von Arten des freien Meeresbodens. Das gleiche gilt auch hinsichtlich des Lichtfaktors. Ferner kann unter dem Gesichtspunkt der Reaktionsweisen der Planula die Temperatur als direkt bestimmender Faktor wohl ebenfalls ausgeschlossen werden, da die Temperaturgradienten im Inneren und außerhalb der Höhlen vermutlich nicht ausgeprägt genug sind.

Wohl aber ist die Temperatur für die Horizontal- und Vertikalverbreitung von ausschlaggebender Bedeutung, da die Phase der Strobilation nach allen Beobachtungen temperaturabhängig ist. Wie schon kurz erwähnt (vgl. p. 437), liegt die obere Grenztemperatur für den Eintritt dieses Prozesses bei etwa $22^{\circ} \mathrm{C}$. Bei konstanten Tempera- 
turen zwischen 17 bis $15^{\circ} \mathrm{C}$ tritt die Strobilation regelmäßig ein, und zwar das ganze Jahr über, so daß die im Meer saisonbedingte Strobilationsperiode nicht genetisch fixiert ist. Bei neuen Versuchen wurden Strobilationen noch bei Temperaturen von 11 bis $12^{\circ} \mathrm{C}$ beobachtet, so daß $S$. eumedusoides nach den für die Fortpflanzung notwendigen Temperaturen als Kälteform angesehen werden muß. Weitere experimentelle Untersuchungen sind notwendig, um diese Aussage zu präzisieren. Immerhin darf nach den bisherigen Ergebnissen die schon früher geäußerte Vermutung als wahrscheinlich gelten, daß S. eumedusoides von Vorfahren abstammt, die in den unteren Bereichen des Sublitoral und des Bathyal gelebt haben (WERNER 1971b, p. 136).

Die abgeänderte Entwicklung durch sessile Medusoide muß nach allem als Anpassung an das spezielle Milieu submariner Höhlen im oberen und mittleren Sublitoral erklärt werden, auch wenn wir uns vorerst mit dieser allgemeinen Aussage begnügen müssen. Dabei kann auf den Parallelfall von $S$. planulophorus hingewiesen werden, bei dem im gleichen Milieu die progressive Evolution im Endeffekt sogar zum völligen Verlust der Medusengeneration geführt hat. In diesem Zusammenhang ist von besonderem Interesse, daß nach RIEDL (1966, p. 511 f.) in submarinen Höhlen der Adria die Hydroidenarten mit sessilen Gonophoren überwiegen.

Wie bei den Hydroiden mit sessilen Gonophoren besteht auch bei S. eumedusoides der ökologisch bedeutsame evolutive Fortschritt in der Abkürzung der pelagischen Phase, die im Leben der marinen Organismen mit freischwimmenden Stadien ja stets die am meisten gefährdete Periode darstellt. Bei den Arten von Stephanoscyphus mit freien Medusen beträgt die Dauer der pelagischen Periode etwa 3 bis 4 Monate; sie ist bei $S$. eumedusoides auf etwa einen Monat verkürzt.

\section{Diagnose}

Stephanoscyphus eumedusoides n. spec.: Solitärer Scyphopolyp mit vollständiger, sehr kräftiger, gelb bis dunkelbraun gefärbter Peridermröhre, die am Substrat mit kleiner Haftscheibe befestigt ist und sich zur oberen Mündung schwach hornförmig erweitert. Röhre sekundär oft unregelmäßig gekrümmt. Oberflächenstruktur mit schmalen, dicht aufeinanderfolgenden Querringen, so daß die Längsstreifung oft undeutlich ist. Zahnbildungen im Inneren der Röhre auf 1 Einzelzahn, selten 2 Einzelzähne im Basalteil reduziert oder fehlend. Basalteil der Röhre oft mit unregelmäßigen, lamellenartigen Wandverstärkungen. Weichkörper mit 30 bis 60 Tentakeln, unterer Rand des Kragenepithels mit gelblichem bis schwach bräunlichem Pigmentring. Perradiale Offnungen des Ringkanals im Kopfteil als Poren ausgebildet. Entwicklung mit typischer Strobilationsphase, durch die in der Polypenröhre eine zusammenhängende Kette von teilweise ineinander geschachtelten sessilen Eumedusoiden entsteht, bei denen wesentliche Medusenmerkmale erhalten sind, deren Morphologie aber charakteristische Reduktionserscheinungen aufweist. Nach der Beschaffenheit der Schirmrandorgane von Vorfahren abstammend, die freie Medusen des Nausithoë-Typs besaßen. Bei den Medusoiden einer Strobilationskette wechselnde Formen von Zwittrigkeit. Entwidklung durch Larviparie: Die Planulae schlüpfen aus den Medusoiden bei deren Zerfall aus, wenn der regenerierende Polyp die Strobilationskette aus der Röhre herausschiebt. 
Dauer der planktischen Phase der Planulae 3 bis 4 Wochen. Bicnidom aus holotrichen Haplonemen und heterotrichen mikrobasischen Eurytelen. Herkunft des Lebendmaterials: submarine Felshöhlen im oberen und mittleren Sublitoral der französischen Mittelmeerküste bei Marseille, konserviertes Material auch aus dem Golf von Neapel, von Tunis und Portugal. Höhlenform.

\section{ZUSAMMENFASSUNG}

1. In submarinen Höhlen der Felsküste von Marseille wurden zahlreiche Exemplare einer neuen solitären Stephanoscyphus-Art gefunden, die in Laboratoriumskultur genommen und über den ganzen Lebenszyklus gezüchtet werden konnte.

2. Morphologie und Lebensgeschichte werden beschrieben. Die neue Art zeichnet sich durch einen für Scyphozoen bisher unbekannten Entwicklungsmodus aus, durch den sie von allen anderen Stephanoscyphus-Arten verschieden ist. Er besteht in der Reduktion der Medusengeneration zu sessilen Medusoiden.

3. Größe, Form und Struktureigenschaften der Peridermröhre des Polypen werden beschrieben. Besondere Merkmale sind die kräftige Beschaffenheit, die Oberflächenstruktur mit dicht aufeinanderfolgenden schmalen Querringen und schwach ausgeprägter oder fehlender Längsstreifung sowie die Reduktion der Zahnbildungen im Inneren. Die Wand des basalen Röhrenteils kann durch zusätzliche unregelmäßige Lamellen verstärkt sein.

4. Habitus und Eigenschaften des Weichkörpers werden beschrieben. Charakteristisch ist das Vorhandensein eines gelblichen oder schwach bräunlichen Pigmentringes in der Epidermis des unteren Kragenrandes.

5. Der Polyp strobiliert in der für Scyphozoen typischen Weise durch Querteilung des Körpers. Die Strobilationsprodukte lösen sich jedoch nicht als freie Ephyren $\mathrm{ab}$, sondern bleiben als sessile Medusoide miteinander und mit dem basalen Restkörper des Polypen in fester Verbindung und werden ohne Zufuhr partikulärer Nahrung geschlechtsreif.

6. Die Morphologie, die Grundzüge der Anatomie und die Entwicklung der Medusoide werden beschrieben. $\mathrm{Da}$ wesentliche Merkmale der Medusenorganisation erhalten sind, können sie als Eumedusoide bezeichnet werden. Gleichzeitig weisen sie charakteristische Reduktionserscheinungen auf, die sekundärer Natur und Ausdruck einer progressiven Evolution sind.

7. Nach der Struktur und Oktomerie der Schirmrandorgane muß angenommen werden, daß die Vorfahren des Polypen freie Medusen des Nausithoë-Typs gehabt haben. Seine frühere Einordnung in ein neues Genus (Tesseroscyphus) muß aufgegeben werden. Der neue Polyp erhält den Namen Stephanoscypbus eumedusoides.

8. Die weitere Entwicklung ist durch die Zwittrigkeit der Medusoide und ihre Larviparie gekennzeichnet. Die befruchteten Eier entwickeln sich in den Gastralräumen zu Planulae. Diese werden am Ende der Fortpflanzungsphase frei, wenn der regenerierende Polyp die Strobilationskette aus der Röhre herausschiebt. Die Kette löst sich dabei in die Einzelmedusoide auf, die absterben und zerfallen. 
9. Durch Isolierungsversuche wurde nachgewiesen, daß Selbstbefruchtung in der gleichen Strobilationskette möglich ist. Doch ist dabei die Fruchtbarkeit, das heißt die Zahl der erzeugten Planulae, verringert.

10. Die freischwimmende Planula heftet sich nach etwa 3-4 Wochen an einem geeigneten Substrat an und wandelt sich in den jungen Polypen um; seine Entwicklung wird kurz beschrieben.

11. Der evolutionistische Fortschritt des neuen Entwicklungsmodus besteht in einer Verlagerung eines wesentlichen Abschnittes der Entwicklung in den Schutz der Peridermröhre und in einer Verkürzung der pelagischen Phase. Diese beträgt bei Arten mit freien Medusen etwa 3 bis 4 Monate und ist bei $S$. eumedusoides auf etwa einen Monat verkürzt.

12. Der Polyp und seine Entwicklungsstadien haben ein Bicnidom aus holotrichen Haplonemen und heterotrichen mikrobasischen Eurytelen.

13. Vertikal- und Horizontalverbreitung werden nach dem jetzigen Stand der Kenntnisse erörtert. In ökologischer Hinsicht ist von Interesse, daß die Strobilation ein temperaturabhängiger Prozeß ist, so daß die Temperatur die Verbreitung begrenzt. Ferner muß S. eumedusoides nach den bisherigen Funden als Höhlenform gelten.

14. In die Artdiagnose müssen die Besonderheiten des Entwicklungsganges aufgenommen werden, da sie die zuverlässige Identifizierung ermöglichen.

Danksagungen: Mein besonderer Dank gilt Herrn Dr. H. Ziвrowrus, Marseille, der die neue Art bei seinen Tauchuntersuchungen entdeckt hat. Seiner unermüdlichen Hilfsbereitschaft und seinem persönlichen Einsatz habe ich es zu verdanken, daß mir ein reichhaltiges Lebendmaterial und auch das konservierte Material zur Verfügung stand, das er bei seinen Untersuchungen über die Scleractinia des Mittelmeeres gefunden hat. Ebenso danke ich dem Direktor der Station Marine d'Endoume, Marseille, Herrn Professor J. M. Pérès, der mir mehrfach Quartier und alle benötigten Hilfsmittel bereitgestellt hat. Danken möchte ich auch Frau A. Reiners und Frl. S. Hogreve, Hamburg, für die Mitarbeit bei den langjährigen Kulturarbeiten. Für die Förderung meiner Arbeiten möchte ich der Deutschen Forschungsgemeinschaft meinen herzlichen Dank aussprechen, die mir die Reisemittel bewilligt hat.

\section{ZITIERTE LITERATUR}

Bhaud, M., Jacques, G. \& Razour, C., 1967. Données météorologiques et hydrologiques de la région de Banyuls-sur-mer. Année 1965 (Point côtier). Vieu Milieu (B) 18, 137-151.

Chapman, D. M. \& Werner, B., 1972. Structure of a solitary and a colonial species of Stephanoscyphus (Scyphozoa, Coronatae) with observations on periderm repair. Helgoländer wiss. Meeresunters. 23, 393-421.

Harckel, E., 1880. Das System der Medusen. I,2: System der Acraspeden. Fischer, Jena, 361-672.

Komar, T., 1935. On Stephanoscyphus and Nausithoë. Mem. Coll. Sci. Kyoto Univ. (B) 10, 289-339.

- 1936. On another form of Stephanoscyphus found in the waters of Japan. Mem. Coll. Sci. Kyoto Univ. (B) 11, 175-183.

- \& Tokuoka, Y., 1939. Further observations on the strobilation of Stephanoscyphus. Mem. Coll. Sci. Kyoto Univ. (B) 15, 127-133.

Kramp, P. L., 1951. Hydrozoa and Scyphozoa. Rep. Swed. deep Sea Exped. 2 (Zool.: 10), 121-127. 
- 1959. Stephanoscyphus (Scyphozoa). Galathea Rep. 1, 173-185.

- 1962. Stephanoscyphus corniformis Komar (Scyphozoa). Lunds Univ. Ārsskr. N.F. (Adv. 2) $58,1-4$.

KüHN, A., 1913. Entwicklungsgeschichte und Verwandtschaftsbeziehungen der Hydrozoen. I. Die Hydroiden. Ergebn. Fortschr. Zool. 4, 1-284.

Lo Bianco, S., 1903. Le pesche abissali eseguite da F. A. Krupr col yadht "Puritan“ nelle adiacenze di Capri ed in altre località del Mediterraneo. Mitt. zool. Stn. Neapel 16, 109-278.

MrNas, H.-J., 1971. Observations hydrologiques dans le golfe de Marseille (Années 1961 à 1965). Téthys 3, 447-457.

Rredl, R., 1966. Biologie der Meereshöhlen. Parey, Hamburg, 636 pp.

WerlL, R., 1934. Contributions à l'étude des cnidaires et de leurs nématocystes. 12. Tray. Stn. zool. Wimereux 10-11, 1-701.

WERNER, B., 1965. Die Nesselkapseln der Cnidaria, mit besonderer Berücksichtigung der Hydroida. I. Klassifikation und Bedeutung für die Systematik und Evolution. Helgoländer wiss. Meeresunters. 12, 1-39.

- 1966. Stephanoscyphus (Scyphozoa, Coronatae) und seine direkte Abstammung von den fossilen Conulata. Helgoländer wiss. Meeresunters. 13, 317-347.

- 1967. Morphologie, Systematik und Lebensgeschichte von Stephanoscyphus (Scyphozoa, Coronatae) sowie seine Bedeutung für die Evolution der Scyphozoa. Zool. Anz. (Suppl.) 30, 297-319.

- 1968. Polypengeneration und Entwicklungsgeschichte von Eucheilota maculata (Thecata, Leptomedusae). Mit einem Beitrag zur Methodik der Kultur mariner Hydroiden. Helgoländer wiss. Meeresunters. 18, 136-168.

- 1970. Weitere Untersuchungen über die Entwicklungsgeschichte von Stephanoscyphus (Scyphozoa, Coronatae) und seine Bedeutung für die Evolution der Scyphozoa. Zool. Anz. (Suppl.) 33, 159-165.

- 1971a. Ein neuer, bisher unbekannter Entwicklungsmodus bei einem Scyphopolypen. Experientia 27, 351-353.

- 1971b. Stephanoscypbus planulophorus n. spec., ein neuer Scyphopolyp mit einem neuen Entwicklungsmodus. Helgoländer wiss. Meeresunters. 22, 120-140.

- 1973. New investigations on systematics and evolution of the class Scyphozoa and the phylum Cnidaria. Publs Seto mar. biol. Lab. 20, 35-61.

Zibrowius, H., 1968. Note préliminaire sur la présence à Marseille de quatre madréporaires peu connus: Desmopbyllum fasciculatum (Risso, 1826), Guynia annulata (Duncan, 1872), Stenocyathus vermiformis (PourTalès, 1868) et Conotrochus magnaghii (CeCCHINI, 1914). Bull. Soc. zool. Fr. 93, 325-330.

Anschrift des Autors: Dr. B. WeRner

Biologische Anstalt Helgoland

(Zentrale)

2 Hamburg 50

Palmaille 9

Bundesrepublik Deutschland 